\title{
A Limit Theorem for Stochastic Acceleration
}

\author{
H. Kesten ${ }^{1}$ and G. C. Papanicolaou ${ }^{2}$ \\ ${ }^{1}$ Department of Mathematics, Cornell University, Ithaca, NY 14853, USA \\ ${ }^{2}$ Courant Institute of Mathematical Sciences, New York University, New York, NY 10012, USA
}

\begin{abstract}
We consider the motion of a particle in a weak mean zero random force field $F$, which depends on the position, $x(t)$, and the velocity, $v(t)=\dot{x}(t)$. The equation of motion is $\ddot{x}(t)=\varepsilon F(x(t), v(t), \omega)$, where $x(\cdot)$ and $v(\cdot)$ take values in $\mathbb{R}^{d}, d \geqq 3$, and $\omega$ ranges over some probability space. We show, under suitable mixing and moment conditions on $F$, that as $\varepsilon \rightarrow 0, v^{\varepsilon}(t) \equiv v\left(t / \varepsilon^{2}\right)$ converges weakly to a diffusion Markov process $v(t)$, and $\varepsilon^{2} x^{\varepsilon}(t)$ converges weakly to $\int_{0}^{t} v(s) d s+x$, where $x=\lim \varepsilon^{2} x^{\varepsilon}(0)$.
\end{abstract}

\section{Introduction}

For simplicity we do not discuss the general situation in this section, but restrict ourselves to force fields which depend on position only.

Let $F(x), x \in \mathbb{R}^{d}$, be a random vector field, a random force field, which is stationary and has mean zero. Let $x(t)$ be the coordinate of a particle of unit mass moving through this force field. The equation of motion is

$$
\ddot{x}=F(x) \text {. }
$$

with given initial position and velocity. Suppose that the force is weak and weakly correlated for points that are far apart. Then one expects that after a long time the velocity $\dot{x}$ will behave like a diffusion Markov process and the position $x$ like the integral of this diffusion process.

To be more specific, suppose that the root mean square of the force field $F$ is proportional to $\varepsilon$ so that we may replace (1.1) by

$$
\ddot{x}=\varepsilon F(x)
$$

in which $F(x)$ is of order one. Rescaling of time $t$ into $t / \varepsilon^{2}$ and putting $\dot{x}\left(t / \varepsilon^{2}\right)=$ $v^{\varepsilon}(t), x\left(t / \varepsilon^{2}\right)=x^{\varepsilon}(t)$ leads from (1.1) to the system.

$$
\begin{aligned}
& \frac{d x^{\varepsilon}(t)}{d t}=\frac{1}{\varepsilon^{2}} v^{\varepsilon}(t) \\
& \frac{d v^{\varepsilon}(t)}{d t}=\frac{1}{\varepsilon} F\left(x^{\varepsilon}(t)\right)
\end{aligned}
$$


It is proved in the following sections that under suitable conditions on $F, v^{\varepsilon}$ converges weakly as $\varepsilon \rightarrow 0$ to a diffusion Markov process $v(t)$ whose generator is given explicitly. Moreover, $\varepsilon^{2} x^{\varepsilon}(t)$ converges weakly to $\int_{0}^{t} v(s) d s+x$, where $x=\lim \varepsilon^{2} x^{\varepsilon}(0)$, as $\varepsilon \rightarrow 0$.

The Eq. (1.2) describes for instance the motion of a charged particle in an electromagnetic field, and several authors have obtained formulas for the limit process by perturbation methods or similar procedures [1-5]. We now give such a formal derivation of the relevant results for (1.3). We note that the method used in [6] for the much simpler problem than (1.3)

$$
\frac{d x^{\varepsilon}(t)}{d t}=\frac{1}{\varepsilon^{2}} v+\frac{1}{\varepsilon} F\left(x^{\varepsilon}(t)\right),
$$

does not work well in the present situation.

Let $f(v)$ be a bounded and smooth function on $\mathbb{R}^{d}$ and let $u^{\varepsilon}(t, x, v)=f\left(v^{\varepsilon}(t ; x, v)\right)$ where $x^{\varepsilon}(t ; x, v), v^{\varepsilon}(t ; x, v)$ is the solution of $(1.3)$ with $x^{\varepsilon}(0)=x, v^{\varepsilon}(0)=v$. As a function of $t, x$ and $v, u^{\varepsilon}$ satisfies (the adjoint) Liouville equation

$$
\begin{aligned}
& \frac{\partial u^{\varepsilon}}{\partial t}=\frac{1}{\varepsilon^{2}} v \cdot \frac{\partial u^{\varepsilon}}{\partial x}+\frac{1}{\varepsilon} F(x) \cdot \frac{\partial u^{\varepsilon}}{\partial v}, \quad t>0, \\
& u^{\varepsilon}(0, x, v)=f(v),
\end{aligned}
$$

Here $\partial / \partial x$ and $\partial / \partial v$ denote the $x$ and $v$ gradient operators and $\cdot$ stands for dot product in $\mathbb{R}^{d}$. We now attempt to solve (1.4) by a formal series expansion $u^{\varepsilon}=$ $u_{0}+\varepsilon u_{1}+\varepsilon^{2} u_{2}+\ldots$ Inserting this into (1.4) and collecting terms leads to the equations

$$
\begin{aligned}
& v \cdot \frac{\partial u_{0}}{\partial x}=0 \\
& v \cdot \frac{\partial u_{1}}{\partial x}+F(x) \cdot \frac{\partial u_{0}}{\partial v}=0 \\
& v \cdot \frac{\partial u_{2}}{\partial x}+F(x) \cdot \frac{\partial u_{1}}{\partial v}-\frac{\partial u_{0}}{\partial t}=0, \text { et. }
\end{aligned}
$$

From (1.5) we conclude that $u_{0}=u_{0}(t, v)$ and $u_{0}(0, v)=f(v)$ (to satisfy (1.4)), but $u_{0}$ is otherwise undetermined at this stage. We consider (1.6) and note that we can write the random function $u_{1}$ in the form

$$
u_{1}(t, x, v)=\chi(x, v) \cdot \frac{\partial u_{0}}{\partial v}
$$

where $\chi(x, v)$ satisfies

$$
v \cdot \frac{\partial \chi}{\partial x}+F(x)=0 .
$$

One may write formally $\chi(x, v)=\int_{0}^{\infty} F(x+v t) d t$ but of course this expression does 
not make sense. We retain it anyway with the understanding that some convergence factor has been introduced (like $\int_{0}^{\infty} e^{-\beta t} F(x+v t) d t$ ).

Now we use this in (1.7) and demand as usual that the expectation of $F \cdot \frac{\partial u_{1}}{\partial v}-\frac{\partial u_{0}}{\partial t}$ be zero. This gives a diffusion equation that determines $u_{0}(t, v)$. Specifically $E u_{0}(t, v)$ satisfies

$$
\frac{\partial E u_{0}}{\partial t}=\mathscr{L} E u_{0}, \quad t>0, \quad u_{0}(0, v)=f(v)
$$

where $\mathscr{L}$ is given by

$$
\begin{aligned}
\mathscr{L} & =\int_{0}^{\infty} E\left\{F(x) \cdot \frac{\partial}{\partial v}\left(F(x+v t) \cdot \frac{\partial}{\partial v}\right)\right\} d t \\
& =\sum_{j, k=1}^{d} \frac{\partial}{\partial v_{j}}\left(A_{j k}(v) \frac{\partial}{\partial v_{k}}\right)
\end{aligned}
$$

with

$$
A_{j k}(v)=\int_{0}^{\infty} E\left\{F_{j}(x) F_{k}(x+v t)\right\} d t .
$$

When the correlations in the force field die out rapidly enough, the diffusion coefficients $A_{j k}(v)$ are well defined if $v \neq 0$ but they are necessarily singular at $v=0$. (Note that $\mathscr{L}$ is not always self-adjoint if $F$ depends on $v$ as well; see (2.3) and (2.4) below.)

The problem then is to show that $v^{\varepsilon}(t)$ converges weakly to the diffusion generated by $\mathscr{L}$ of (1.11) under some suitable hypotheses. The theorem of the next section gives such conditions for convergence. It is discussed further there. Some specific examples are given in Sect. 4.

It is of interest to point out some special cases of (1.11) and (1.12) here.

Let

$$
R_{j k}(x)=E\left\{F_{j}(x+y) F_{k}(y)\right\}, \quad j, k=1,2, \ldots, d,
$$

be the covariance of the force field $F$. It is assumed that it decays rapidly with $x$; in fact much stronger asymptotic independence assumptions are introduced in the next section. Let us assume also that the symmetry condition

$$
R_{j k}(x)=R_{j k}(-x)
$$

holds. Then (1.12) may be written in the form

$$
A_{j k}(v)=a_{j k}(v) \equiv \frac{1}{2} \int_{-\infty}^{\infty} R_{j k}(v t) d t .
$$

If we introduce the power spectral density $\hat{R}_{j k}(l)$, then

$$
R_{j k}(x)=\int_{\mathbb{R}^{d}} e^{i l . x} \hat{R}_{j k}(l) d l,
$$


and (1.15) becomes

$$
a_{j k}(v)=\pi \int_{\mathbb{R}^{d}} \delta(l \cdot v) \hat{R}_{j k}(l) d l
$$

where $\delta(\alpha)$ is the delta function with unit mass at zero. The expression (1.17) for the diffusion coefficients is useful when $F(x)$ is the gradient of a potential $V(x)$. If we set

$$
R(x-y)=E\{V(x) V(y)\}
$$

then (1.17) yields

$$
\begin{aligned}
a_{j k}(v) & =\pi \int_{\mathbb{R}^{d}} \delta(l \cdot v) l_{j} l_{k} \hat{R}(l) d l \\
& =\sum_{p, q}\left(\delta_{p j}-\frac{v_{p} v_{j}}{|v|^{2}}\right)\left(\delta_{q k}-\frac{v_{q} v_{k}}{|v|^{2}}\right) \pi \int_{\mathbb{R}^{d}} \delta(l \cdot v) l_{p} l_{q} \hat{R}(l) d l .
\end{aligned}
$$

From this we see that in the potential case the limiting diffusion operator is degenerate. The limit diffusion process is concentrated on the sphere $|v|=\left|v_{0}\right|$ where $v_{0} \neq 0$ is the starting velocity. (Since $a(v) \cdot v=0$ and $L\left(v^{2}\right)=0 \cdot$ ) (2.6) is automatic when $|v|$ is constant, but unfortunately, our theorem as stated does not allow $a_{i j}(v)$ to become singular, and hence does not apply without modification to the above case. One such modification of the theorem is given in Remark 5 of Section 4. The conclusion of the theorem remains valid if (4.2) and (4.3) hold, even when $a(\cdot)$ becomes singular. This comment also applies to other cases where $|v(t)|$ remains constant (e.g. When $F(x, v)$ is always perpendicular to $v$, such as when $F(x, v)$ is of the form $F(x, v)=v \wedge \Gamma(x))$.

\section{Statement of Theorem}

Throughout $(\Omega, \mathscr{F}, \mathscr{P})$ denotes our basic probability space. On this space $F(x, v, \omega)$ : $\mathbb{R}^{d} \times \mathbb{R}^{d} \times \Omega \rightarrow \mathbb{R}^{d}$ is a random field with the following properties:

(I) $F$ is jointly measurable with respect to $\mathscr{B} \times \mathscr{B} \times \mathscr{F}$ where $\mathscr{B}$ is the Borel field in $\mathbb{R}^{d}$. As a function of $(x, v), F(\cdot, \omega)$ is almost surely in $C^{2}\left(\mathbb{R}^{d} \times \mathbb{R}^{d}\right)$.

(II) $F$ is strictly stationary in $x$, i.e. for any $x_{i}, v_{i} \in \mathbb{R}^{d}$ the joint distribution of $F\left(x_{1}+h, v_{1}\right), \ldots, F\left(x_{k}+h, v_{k}\right)$ is independent of $h \in \mathbb{R}^{d}$. Equivalently, the process $\{F(x, \cdot, \omega)\}_{x \in \mathbb{R}^{d}}$ is stationary in $x$. In addition

$$
E\{F(x, v)\}=0, \quad x, v \in \mathbb{R}^{d} .
$$

(III) For $\Lambda \subset \mathbb{R}^{d}$, set

$$
\begin{aligned}
\mathscr{G}_{\Lambda} & =\sigma\left\{F(x, v, \cdot): x \in \Lambda, v \in \mathbb{R}^{d}\right\} \\
& =\operatorname{sigma} \text { field generated by } F(x, v, \cdot), x \in \Lambda, v \in \mathbb{R}^{d} .
\end{aligned}
$$

For $\Lambda_{1}, \Lambda_{2} \subset \mathbb{R}^{d}$ define

$$
\alpha\left(\Lambda_{1}, \Lambda_{2}\right)=\sup _{A \in \mathscr{G}_{\Lambda_{1}, B \in \mathscr{G}_{\Lambda_{2}}}}|P(B)-P(B \mid A)|
$$

Also, set

$$
\beta(\rho)=\sup \left\{\alpha\left(\Lambda_{1}, \Lambda_{2}\right): \Lambda_{1}, \Lambda_{2} \in \mathscr{B} \text { with } d\left(\Lambda_{1}, \Lambda_{2}\right) \geqq \rho\right\} .
$$


Here

$$
d\left(\Lambda_{1}, \Lambda_{2}\right)=\inf \left\{\left|x_{1}-x_{2}\right|: x_{i} \in \Lambda_{i}\right\} .
$$

Assume that

$$
\int_{0}^{\infty}\{\beta(t)\}^{1 / 24} d t<\infty
$$

Note that (2.2) is in a sense a measure of the rate at which $F\left(x_{1}, \cdot\right)$ and $F\left(x_{2}, \cdot\right)$ become independent when $\left|x_{2}-x_{1}\right| \rightarrow \infty$.

(IV) For some constant $C_{0}$ and all $v_{0} \in \mathbb{R}^{d}, 0 \leqq|\beta| \leqq 2$ and $r=16 d+64$

$$
E\left\{\sup _{|x| \leqq 1,\left|v-v_{0}\right| \leqq 1}\left|D^{\beta} F(x, v)\right|^{r}\right\} \leqq C_{0} .
$$

As usual $\beta$ stands here for a multi-index and $D^{\beta}$ for the corresponding partial derivative. Thus $D^{\beta} F(x, v)$ can be any mixed derivative of $F$.

(V) Let

$$
\begin{aligned}
a_{i j}(v)= & \int_{-\infty}^{+\infty} E\left\{F_{i}(0, v) F_{j}(t v, v)\right\} d t, \\
b_{i}(v)= & \sum_{j} \int_{0}^{\infty} E\left\{F_{j}(0, v) \frac{d}{d v_{j}} F_{i}(t v, v)\right\} d t \\
= & \sum_{j} \int_{0}^{\infty}\left[t E\left\{F_{j}(0, v) \frac{\partial}{\partial x_{j}} F_{i}(t v, v)\right\}\right. \\
& \left.+E\left\{F_{j}(0, v) \frac{\partial}{\partial v_{j}} F_{i}(t v, v)\right\}\right] d t .
\end{aligned}
$$

Here $\left(\partial / \partial v_{j}\right) F(t v, v)=\left[\left(\partial / \partial v_{j}\right) F(x, v)\right]_{x=t v}$. The integrals in (2.3) and (2.4) can be shown to be absolutely convergent on the set $\{v \neq 0\}$ and to be bounded as $|v| \rightarrow \infty$ by means of (III) and (IV) (use Theorem 17.23 of [7] or Lemma 20.1 of [8]). Assume that $a_{i j}(v)$ is strictly positive definite on $\{v \neq 0\}$ and that $a_{i j}(\cdot)$ and $b_{i}(\cdot)$ are $C^{\infty}$ functions on $\{v \neq 0\}$.

For any $f \in C^{2}\left(\mathbb{R}^{d}\right)$ define

$$
\mathscr{L} f(v)=\frac{1}{2} \sum_{i, j} a_{i j}(v) \frac{\partial^{2}}{\partial v_{i} \partial v_{j}} f(v)+\sum_{i} b_{i}(v) \frac{\partial f}{\partial v_{i}}, v \neq 0 .
$$

Let $V_{t}$ be a diffusion with generator $\mathscr{L}$ and starting-point $v_{0} \neq 0$ (see Remark 1 below). Assume finally that for each $v_{0} \neq 0$ and $T<\infty$

$$
\lim _{M \rightarrow \infty} P^{v_{0}}\left\{\left|V_{t}\right| \leqq \frac{1}{M} \text { for some } t \leqq T\right\}=0
$$


Last, let $\left\{v^{\varepsilon}(t), y^{\varepsilon}(t)\right\}=\left\{v^{\varepsilon}(t, \omega), y^{\varepsilon}(t, \omega)\right\}$ be the solution of the equations

$$
\begin{aligned}
\frac{d y^{\varepsilon}}{d t} & =\frac{1}{\varepsilon^{2}} v^{\varepsilon}(t) \\
\frac{d v^{\varepsilon}}{d t} & =\frac{1}{\varepsilon} F\left(y^{\varepsilon}(t), v^{\varepsilon}(t)\right) \\
v^{\varepsilon}(0) & =v_{0} \neq 0, y^{\varepsilon}(0)=y_{0} .
\end{aligned}
$$

These solutions exist and are unique with probability one by the argument in step (ii) of [6]. Denote by $Q^{\varepsilon}$ the probability measure on $C=C\left([0, \infty) ; \mathbb{R}^{d}\right)$ induced by $\left\{v^{\varepsilon}(t)\right\}_{t \geqq 0}$.

Theorem. If $d \geqq 3, v_{0} \neq 0$ and $F(\cdot)$ satisfies conditions $(I)-(V)$ above, then $Q^{\varepsilon}$ converges weakly on $C$ as $\varepsilon \downarrow 0$ to the measure $Q$ corresponding to the diffusion process in $\mathbb{R}^{d}$ with generator $\mathscr{L}$ and initial point $v_{0}\left(\right.$ i.e., $\left.Q\left(v(0)=v_{0}\right)=1\right)$.

Corollary. Under the conditions of the theorem the measure $R^{\varepsilon}$ induced by $\left(v^{\varepsilon}(\cdot), \varepsilon^{2} y^{\varepsilon}(\cdot)\right)$ on $C\left([0, \infty) ; \mathbb{R}^{d} \times \mathbb{R}^{d}\right)$ converges weakly to the unique measure $R$ which is concentrated on the set

$$
\left\{X, Y: Y_{i}(t)=\int_{0}^{t} X_{i}(\sigma) d \sigma, X(0)=v_{0}\right\}
$$

and whose marginal distribution of $X(\cdot)$ coincides with $Q$.

(Here $X_{1}(t), \ldots, X_{d}(t), Y_{1}(t), \ldots, Y_{d}(t)$ are the coordinate functions on $C\left([0, \infty) ; \mathbb{R}^{d} \times \mathbb{R}^{d}\right)$.

Remark 1. The diffusion $V_{t}$ on $\mathbb{R}^{d} \backslash\{0\}$ can be constructed by "patching together" local diffusions. The local diffusions can be obtained as solutions of suitable Ito equations (see [9], Ch. 4.3) or by semigroup theory (see [10]). It is also possible to define the diffusion $V_{t}^{(n)}$ which has generator $\mathscr{L}$ on

$$
C_{n}=\left\{v \in \mathbb{R}^{d}:|v|>\frac{1}{n}\right\},
$$

and is killed at time $\tau_{n}=\inf \left\{t: V_{t}^{(n)} \notin C_{n}\right\}$. For $m \geqq n, V_{t}^{(m)}$ up until time $\tau_{n}$ is equivalent to $V_{t}^{(n)}\left([11]\right.$, Corollary in Chap. 5.24), and $V_{t}$ can be viewed as a limit of the $V_{t}^{(n)}$.

Remark 2. In our most important examples (see Remark 3) the coefficients $a_{i j}(v)$ and $v_{i}(v)$ are singular at the origin so that one should not replace $(V)$ by the simpler condition $a_{i j}(\cdot), b_{i}(\cdot) \in C^{\infty}\left(\mathbb{R}^{d}\right)$.

In Remark 6, Sect. 4, we shall discuss a replacement for the condition $d \geqq 3$ and $a_{i j}(\cdot), b_{i}(\cdot) \in C^{\infty}\left(\mathbb{R}^{d} \backslash\{0\}\right)$. We shall also give some sufficient conditions for (2.6). For the definition of the spaces $C\left([0, \infty) ; \mathbb{R}^{d}\right), D\left([0, \infty) ; \mathbb{R}^{d}\right)$ and weak convergence on these spaces see [12] and [13].

Remark 3. Note that under $R$ the process $\{X(t), Y(t)\}_{t \geqq 0}$ of the corollary is a singular diffusion; the $Y$-part has zero diffusion coefficients. By itself the $Y$-part is not Markovian, let alone a diffusion. 


\section{Proof of Theorem}

The basic outline of the proof is the same as for Theorem 3 of [6]. We first introduce a truncated process (in step (i)). The truncation will be removed only in the last step. The second step proves the basic mixing lemma which is used in step (iii) to show tightness of the family of measures (indexed by $\varepsilon$ ) induced by the truncated processes. The remaining steps identify the limit process as the solution of a certain martingle problem.

\section{Step $(i)$}

In contrast to [6] we need here not one, but several cutoff functions. These will depend on parameters $\eta, \delta, M, N$, which remain fixed until step (v). We shall not exhibit these parameters explicitly in the notation before step (v); it is understood, though, that all constants $C$ below may depend on these parameters, the dimension, $d$, and the length of the time interval, $T$, but not on $\varepsilon$.

As will become apparent it is best to define the cutoff functions as nonanticipatory functionals which depend in addition on a variable which ranges over $\mathbb{R}^{d}$. We begin with the velocity cutoff. Let $D=D\left([0, \infty) ; \mathbb{R}^{d}\right)$ and $\eta>0$, and for $X(\cdot) \in D$ set

$$
X_{k}= \begin{cases}\frac{X(k \eta)}{|X(k \eta)|} & \text { if } X(k \eta) \neq 0 \\ (1,0, \ldots, 0) & \text { if } X(k \eta)=0 .\end{cases}
$$

In addition let $\psi_{0}: \mathbb{R}^{d} \times S^{d-1} \times S^{d-1} \rightarrow[0,1]$ be a $C^{\infty}$ function $\left(S^{d-1}\right.$ is the unit ball in $\mathbb{R}^{d}$ ) such that

$$
\begin{aligned}
& \psi_{0}\left(u, x_{1}, x_{2}\right)=0 \quad \text { if } \quad|u| \leqq \frac{1}{2 M} \text { or }|u| \geqq 2 M \\
& \text { or }\left(u, x_{1}\right) \leqq \frac{1}{2 N} \quad \text { or } \quad\left(u, x_{2}\right) \leqq \frac{1}{2 N} \\
& \psi_{0}\left(u, x_{1}, x_{2}\right)=1 \quad \text { if } \quad \frac{1}{M} \leqq|u| \leqq M \quad \text { and } \\
& \left(u, x_{1}\right) \geqq \frac{1}{N} \text { and }\left(u, x_{2}\right) \geqq \frac{1}{N} .
\end{aligned}
$$

Throughout we take $M$ so large that

$$
\frac{1}{M} \leqq\left|v_{0}\right| \leqq M
$$

Now define $\Psi:[0, \infty) \times D \times \mathbb{R}^{d}$ by

$$
\Psi(t, X, w)=\left\{\begin{array}{lll}
\psi_{0}\left(w, X_{0}, X_{0}\right) & \text { if } \quad 0 \leqq t<\eta \\
\psi_{0}\left(w, X_{k-1}, X_{k}\right) & \text { if } \quad k \eta \leqq t<(k+1) \eta, k \geqq 1
\end{array}\right.
$$


To prevent the $y^{\varepsilon}$ path at any given time to come too close to a value taken on before another cutoff function is needed. It will be seen in step (ii) how this guarantees a certain amount of independence between the present and the "distant past" for the truncated process, and thereby allows us to prove the mixing lemma. We construct a function $\phi_{k}: D \times \mathbb{R}^{d} \rightarrow[0,1]$ which is smooth in its second argument, uniformly in the first argument and $k$. The principal requirement for $\phi_{k}$ if $k \geqq 1$ is that for fixed $X(\cdot) \in D$,

$$
\begin{array}{lcc}
\phi_{k}(X, z)=0 \quad \text { if } & \inf _{0 \leqq u \leqq(k-1) \eta}\left|z-\int_{0}^{u} X(t) d t\right| \leqq \delta, \\
\phi_{k}(X, z)=1 \quad \text { if } & \inf _{0 \leqq u \leqq(k-1) \eta}\left|z-\int_{0}^{u} X(t) d t\right| \geqq 2 \delta .
\end{array}
$$

To construct such a function we take

$$
\chi_{k}(X, z)=\tilde{\chi}\left(\inf _{0 \leqq u \leqq(k-1) \eta}\left|z-\int_{0}^{u} X(t) d t\right|\right)
$$

where $\tilde{\chi}$ is continuous, $0 \leqq \tilde{\chi} \leqq 1$ and

$$
\tilde{\chi}(y)=\left\{\begin{array}{lll}
0 & \text { if } & |y| \leqq \frac{5}{4} \delta \\
1 & \text { if } & |y| \geqq \frac{7}{4} \delta .
\end{array}\right.
$$

Also we take for $\Delta(\cdot)$ a nonnegative function $C^{\infty}\left(\mathbb{R}^{d}\right)$ with support in $\{|z| \leqq \delta / 4\}$ and such that

$$
\int_{\mathbb{R}^{d}} \Delta(z) d z=1 .
$$

Then

$$
\phi_{k}(X, z)=\int_{\mathbb{R}^{d}} \Delta(z-x) \chi_{k}(X, x) d x
$$

satisfies (3.4). Finally we define $\Phi=\Phi_{\varepsilon}:[0, \infty) \times D \times \mathbb{R}^{d}$ by

$$
\begin{array}{ll}
\Phi(t, X, z) \equiv 1 & \text { if } 0 \leqq t<\eta, \\
\Phi(t, X, z)=\phi_{k}\left(X, \varepsilon^{2}\left(z-y_{0}\right)\right) & \text { if } k \eta \leqq t<(k+1) \eta, k \geqq 1 .
\end{array}
$$

Lastly, we set

$$
G(t, X, z, w)=G_{\varepsilon}(t, X, z, w)=\Psi(t, X, w) \Phi_{\varepsilon}(t, X, z) F(z, w)
$$

and we define our truncated process $u(\cdot), z(\cdot)$ as the solution of

$$
\begin{aligned}
\frac{d z}{d t} & =\frac{1}{\varepsilon^{2}} w(t) \\
\frac{d w}{d t} & =\frac{1}{\varepsilon} G(t, w(\cdot), z(t), w(t)) \\
w(0) & =v_{0}, \quad z(0)=y_{0} .
\end{aligned}
$$


As in [6] this means that $z$ and $w$ are continuous functions which satisfy

$$
\begin{aligned}
& z(t)=y_{0}+\frac{1}{\varepsilon^{2}} \int_{0}^{t} w(\sigma) d \sigma \\
& w(t)=v_{0}+\frac{1}{\varepsilon} \int_{0}^{t} G(\sigma, w(\cdot), z(\sigma), w(\sigma)) d \sigma
\end{aligned}
$$

Note that $G$ is continuously differentiable in its last two arguments and for $k \eta \leqq t<(k+1) \eta$ depends on $w(\cdot)$ only through the values of $w(u)$ on $u \leqq k \eta$. In particular, for $t<\eta, G$ does not depend on its second argument and (3.9) has w.p. 1 a unique solution on $t \leqq \eta$ by the argument of step (ii) of [6]. Once a solution has been found for $t \leqq k \eta$, the dependence of $G$ on its second argument is determined up to time $(k+1) \eta$ and by step (ii) of [6] one then obtains w.p. 1 a unique solution for $t \leqq(k+1) \eta$.

Of course $w(\cdot)$ and $z(\cdot)$ depend on $\varepsilon$. When necessary we shall indicate this by writing $w^{\varepsilon}(t)$ and $z^{\varepsilon}(\mathrm{t})$. In particular we denote by $R^{\varepsilon}$ the measure induced on $C\left([0, \infty) ; \mathbb{R}^{d}\right)$ or $D=D\left([0, \infty) ; \mathbb{R}^{d}\right)$ by $w^{\varepsilon}(\cdot)$. Towards the end we shall write $R^{\varepsilon}(\cdot ; M, N, \eta, \delta)$ to indicate the dependence of $R^{\varepsilon}$ on $M, N, \eta, \delta$. For brevity we shall write

$$
G(t, w, z)=G(t, w(\cdot), w, z)
$$

for $w, z \in \mathbb{R}^{d}$ and $w(\cdot)$ the solution of (3.8). Similarly

$$
\Psi(t, w)=\Psi(t, w(\cdot), w), \quad \Phi(t, z)=\Phi(t, w(\cdot), z) .
$$

Before turning to the proof of tightness of the family of measures $\left\{R^{\varepsilon}(): 0<\varepsilon \leqq 1\right\}$ we need some simple observations. First, $G(t, w, z)$ is constant in $t$ over each of the intervals $[k \eta,(k+1) \eta)$. Second, for every $T$ there exists a constant $C_{1}=C_{1}(T, M, \eta, \delta)$ such that

$$
\left|\left(\frac{\partial}{\partial z}\right)^{\beta} \Phi(t, X, z)\right| \leqq C_{1} \varepsilon^{2|\beta|},\left|\left(\frac{\partial}{\partial w}\right)^{\beta} \Psi(t, X, w)\right| \leqq C_{1}
$$

for all $X \in D, w, z \in \mathbb{R}^{d}, 0 \leqq t \leqq T, 0<\varepsilon \leqq 1$ and $|\beta| \leqq 1$. Formula (3.10) is obvious for $\Psi$ from (3.3); for $\Phi$ it follows from (3.5) and (3.6). Indeed, for $k \eta \leqq t<(k+1) \eta$,

$$
\begin{aligned}
\left|\left(\frac{\partial}{\partial z}\right)^{\beta} \Phi(t, X, z)\right| & =\varepsilon^{2|\beta|}\left|\int_{\mathbb{R}^{d}} D^{\beta} \Delta\left(\varepsilon^{2}\left(z-y_{0}\right)-x\right) \chi_{k}(X, x) d x\right| \\
& \leqq \varepsilon^{2|\beta|} \int_{\mathbb{R}^{d}}\left|D^{\beta} \Delta(x)\right| d x .
\end{aligned}
$$

Lastly, for any $z$ of the form $z(\xi)+\frac{\sigma-\xi}{\varepsilon^{2}} w(\xi), \Phi_{\varepsilon}(t, z)$ does not depend explicitly on $\varepsilon$, but only through $\left\{w(u): 0 \leqq u \leqq \xi \vee((k-1) \eta)^{+}\right\}$, when $k \eta \leqq t<(k+1) \eta$. 
Indeed for such $t$, the above $z$ and $u \leqq((k-1) \eta)^{+}$

$$
\begin{aligned}
& \varepsilon^{2}\left\{z(\xi)+\frac{\sigma-\xi}{\varepsilon^{2}} w(\xi)-y_{0}\right\}-\int_{0}^{u} w(t) d t \\
& =\int_{u}^{\xi} w(\lambda) d \lambda+(\sigma-\xi) w(\xi) .
\end{aligned}
$$

Finally, we have the following simple

\section{Lemma 1.}

$$
\begin{aligned}
& \frac{1}{2 M} \leqq|w(t)| \leqq 2 M \quad \text { for all } t \geqq 0 . \\
& \left(w(t), w_{k-1}\right) \geqq \frac{1}{2 N} \quad \text { and }\left(w(t), w_{k}\right) \geqq \frac{1}{2 N} \\
& \quad \text { for } k \eta \leqq t<(k+1) \eta,
\end{aligned}
$$

where $w_{j}=\frac{w(j \eta)}{|w(j \eta)|}, \quad\left(w_{-1}=w_{0}\right)$

$$
\begin{gathered}
|z(t)-z(s)| \leqq \frac{2 M}{\varepsilon^{2}}|t-s|, \quad t, s \geqq 0 \\
P\left\{|w(t)-w(s)| \geqq|t-s| \varepsilon^{-9 / 8} \quad \text { for some } 0 \leqq s, t \leqq T\right\} \leqq C_{2} \varepsilon^{8}
\end{gathered}
$$

Proof. Formulas (3.11) and (3.12) are easily proved by induction on $k$. If they hold at $t=k \eta$, then they must hold up till $(k+1) \eta$ because $\Psi(t, w(t))$ vanishes as soon as (3.11) or (3.12) fails. Formula (3.13) is immediate from (3.9) and (3.11). Lastly, for (3.14) observe that

$$
\begin{gathered}
|w(t)-w(s)|=\frac{1}{\varepsilon}\left|\int_{s}^{t} G(\lambda, z(\lambda), w(\lambda)) d \lambda\right| \\
\leqq \frac{|t-s|}{\varepsilon} \sup _{|\lambda| \leqq T}|F(z(\lambda), w(\lambda))|
\end{gathered}
$$

so that by (3.11), (3.13)

$$
\begin{aligned}
& \leqq P\left\{|w(t)-w(s)| \geqq|t-s| \varepsilon^{-9 / 8} \text { for some } 0 \leqq s, t \leqq T\right\} \\
& \leqq P\left\{\sup _{|\lambda|}|F(z(\lambda), w(\lambda))| \geqq \varepsilon^{-1 / 8}\right\} \\
& \leqq P\left\{\begin{array}{l}
|\lambda| \leqq T \\
\left.\sup _{|z| \leqq\left(2 M / \varepsilon^{2}\right) T+\left|y_{0}\right|,|w| \leqq 2 M}|F(z)| \geqq \varepsilon^{-1 / 8}\right\} .
\end{array}\right.
\end{aligned}
$$

Formula (3.14) now follows from the fact that the set $\left\{(z, w):|z| \leqq\left(2 M / \varepsilon^{2}\right) T+\left|y_{0}\right|\right.$, $|w| \leqq 2 M\}$ can be covered by at most $C_{3}\left((T+1) / \varepsilon^{2}\right)^{d}$ cubes of edge-length one, 
and for any $z_{0}, w_{0}$

$$
\begin{aligned}
& P\left\{\sup _{\left|z-z_{0}\right| \leqq 1,\left|w-w_{0}\right| \leqq 1}|F(z, w)| \geqq \varepsilon^{-1 / 8}\right\} \\
& \quad \leqq \varepsilon^{r / 8} E\left\{\sup _{\left|z-z_{0}\right| \leqq 1,\left|w-w_{0}\right| \leqq 1}|\mathrm{~F}(\mathrm{z}, \mathrm{w})|^{r}\right\} \leqq \varepsilon^{r / 8} C_{0}
\end{aligned}
$$

(by (II) and (IV)). Thus, the left-hand side of (3.14) is at most

$$
\begin{aligned}
C_{3}\left(\frac{T+1}{\varepsilon^{2}}\right)^{d} \varepsilon^{r / 8} C_{0} & =C_{0} C_{3}(T+1)^{d} \varepsilon^{r / 8-2 d} \\
& =C_{0} C_{3}(T+1)^{d} \varepsilon^{8} .
\end{aligned}
$$

\section{Step (ii)}

This is devoted to the fundamental mixing Lemma 4 and some of its consequences. The preparatory Lemma 3 gives a bound for expectations along the path of $z$ which will be used frequently. Both lemmas rely on the possibility of "predicting" $z(\sigma)$ by the linear function $z(\xi)+\left((\sigma-\xi) / \varepsilon^{2}\right) w(\xi)$, which depends only on the path up until time $\xi$. A crucial role is also played by the measure theoretical Lemma 2 which follows directly from the definition of the mixing coefficient $\beta$. For convenience we extend the definition of $\beta$ by setting

$$
\beta(\rho)=2 \text { for } \rho \leqq 0
$$

We also replace $\beta(\cdot)$ by its left continuous modification. This can always be done without invalidating (III) because $\beta(\cdot)$ is nonincreasing. We need a further convention. For $\theta=\left(\theta^{\prime}, \theta^{\prime \prime}\right) \in \mathbb{R}^{d} \times \mathbb{R}^{d}, \tau_{\theta} F$ will denote the random field whose value at $(z, w)$ is given by

$$
\tau_{\theta} F(z, w)=F\left(z+\theta^{\prime}, w+\theta^{\prime \prime}\right)
$$

If $h$ is a function of the $F(z, w)$ which depends only on $\left\{F(z, u): z \in \Lambda, w \in \mathbb{R}^{d}\right\}$ and such that $h(F)$ is $\mathscr{G}_{\Lambda}$ measurable for some $\Lambda \subset \mathbb{R}^{d}$, then we see immediately that $h\left(\tau_{\theta} F\right)$ is $\mathscr{G}_{\Lambda+\theta^{\prime}}$ measurable. In the next lemma we shall take $\theta$ itself also random. Lastly, we set

$$
\mathscr{F}_{t}=\sigma\{z(u), w(u), F(z(u), w(u)): u \leqq t\}
$$

Lemma 2. Let $X$ be an $\mathscr{F}_{t}$ measurable random variable with $E\{|X|\}<\infty$ and let $g_{i}=\left(g_{i}^{\prime}, g_{i}^{\prime \prime}\right)$ be $\mathbb{R}^{d} \times \mathbb{R}^{d}$ valued random variables, measurable with respect to $\mathscr{F}_{t}$ and such that

$$
\min \left\{\left|g_{i}^{\prime}-z(u)\right|: u \leqq t\right\} \geqq \rho
$$

a.e. on the set $\{X \neq 0\}$. Lastly, let $h_{i}$ be Borel functions of $\left\{F(z, w): z \in \Delta_{i}, w \in \mathbb{R}^{d}\right\}$ for Borel sets $\Delta_{i} \subset \mathbb{R}^{d}$ with

$$
0 \in \Delta_{i}, \text { diameter } \Delta_{i} \leqq \kappa
$$

and

$$
\left|h_{i}(F)\right| \leqq A \text { everywhere. }
$$


For $\theta_{i} \in \mathbb{R}^{d} \times \mathbb{R}^{d}$ set

$$
U_{i}(\theta)=E\left\{h_{i}\left(\tau_{\theta} F\right)\right\}, \quad V\left(\theta_{1}, \theta_{2}\right)=E\left\{h_{1}\left(\theta_{1}\right) h_{2}\left(\theta_{2}\right)\right\} .
$$

Then

$$
\left|E\left\{X h_{1}\left(\tau_{g_{1}} F\right)\right\}-E\left\{X U_{1}\left(g_{1}\right)\right\}\right| \leqq 2 A \beta(\rho-\kappa) E\{|X|\},
$$

and

$$
\left|E\left\{X h_{1}\left(\tau_{g_{1}} F\right) h_{2}\left(\tau_{g_{2}} F\right)\right\}-E\left\{X V\left(g_{1}, g_{2}\right)\right\}\right| \leqq 2 A^{2} \beta(\rho-\kappa) E\{|X|\} .
$$

If $(3.18)$ is replaced by

$$
\min \left\{\left|g_{2}^{\prime}-z(u)\right|: u \leqq t\right\} \geqq \rho \text { and }\left|g_{2}^{\prime}-g_{1}^{\prime}\right| \geqq \rho
$$

a.e. on the set $\{X \neq 0\}$, then

$$
\begin{aligned}
& \left|E\left\{X h_{1}\left(\tau_{g_{1}} F\right) h_{2}\left(\tau_{g_{2}} F\right)\right\}-E\left\{X h_{1}\left(\tau_{g_{1}} F\right) U_{2}\left(g_{2}\right)\right\}\right| \\
& \quad \leqq 2 A^{2} \beta(\rho-2 \kappa) E\{|X|\} .
\end{aligned}
$$

Proof. We only prove (3.21). First we change $g_{1}, g_{2}$ on the set $X=0$ such that (3.18) holds everywhere. Since $\{X=0\} \in \mathscr{F}_{t}$ we can do this in such a way that the modified $g_{i}$ are still $\mathscr{F}_{t}$ measurable. Moreover this modification does not affect (3.21). We may also assume $\rho-\kappa>0$ since we took $\beta(\rho)=2$ for $\rho \leqq 0$. Now take $0<\tau<(\rho-\kappa) / 2$ and let $C_{1}, C_{2}, \ldots$ be a sequence of disjoint cubes whose union is all of $\mathbb{R}^{d}$ and such that diameter $\left(C_{i}\right) \leqq \tau$. Let

$$
\begin{aligned}
& D_{i}=\left\{z: d\left(z, C_{i}\right) \geqq \rho-\tau\right\} \\
& E_{i}=\left\{z: d\left(z, C_{i}\right)>\rho-2 \tau\right\} \\
& I_{i}=\text { indicator function of }\left\{g_{1}^{\prime} \in C_{i}\right\},
\end{aligned}
$$

and last,

$$
R=\{z(u): u \leqq t\} .
$$

$R$ is the (random) range of $z(\cdot)$ up until time $t$, and it follows from (3.18) that if $g_{1}^{\prime} \in C_{i}$, then $R$ must be contained in $D_{i}$. Consequently

$$
E\left\{X h_{1}\left(\tau_{g_{1}} F\right)\right\}=\sum_{i} E\left\{X h_{1}\left(\tau_{g_{1}} F\right) I_{i}\right\}=\sum_{i} E\left\{X h_{1}\left(\tau_{g_{1}} F\right) I_{i} ; R \subset D_{i}\right\} .
$$

Now $E_{i}$ is an open neighborhood of $D_{i}$, and we proceed to show that for any $\mathscr{F}_{t}$ measurable random variable $Y$ one has

$$
Y I\left[R \subset D_{i}\right] \text { is } \mathscr{G}_{E_{i}} \text { measurable. }
$$

To verify (3.26) it suffices to consider only $Y$ 's of the form

$$
Y=\prod_{j=1}^{l} K_{j}\left(z\left(u_{j}\right), w\left(u_{j}\right), F\left(z\left(u_{j}\right), w\left(u_{j}\right)\right)\right)
$$

with $0 \leqq u_{j} \leqq t$ and $K_{j}: \mathbb{R}^{d} \times \mathbb{R}^{d} \times \mathbb{R}^{d} \rightarrow \mathbb{R}^{d}$ bounded Borel functions (e.g. by [14], 
Theorem I.20). Now let $\zeta: \mathbb{R}^{d} \rightarrow[0,1]$ be a smooth function such that

$$
\zeta(z)=\left\{\begin{array}{lll}
1 & \text { if } & d\left(z, C_{i}\right) \geqq \rho-\frac{3 \tau}{2} \\
0 & \text { if } & z \notin E_{i},
\end{array}\right.
$$

and set

$$
G^{*}(t, w(\cdot), z, w)=G(t, w(\cdot), z, w) \zeta(z) .
$$

Also, let $z^{*}(\cdot), w^{*}(\cdot)$ be the solution of

$$
\begin{aligned}
& z^{*}(t)=y_{0}+\frac{1}{\varepsilon^{2}} \int_{0}^{t} w^{*}(\sigma) d \sigma \\
& w^{*}(t)=v_{0}+\frac{1}{\varepsilon} \int_{0}^{t} G^{*}\left(\sigma, w^{*}(\cdot), z^{*}(\sigma), w^{*}(\sigma)\right) d \sigma
\end{aligned}
$$

One can obtain $z^{*}, w^{*}$ by the usual iteration procedure, i.e. $z^{*}, u^{*}=\lim _{n \rightarrow \infty}\left(z^{(n)}, u^{(n)}\right)$, where $z^{(0)}(t) \equiv y_{0}, w^{(0)}(t) \equiv v_{0}$ and

$$
\begin{aligned}
z^{(n+1)}(t) & =y_{0}+\frac{1}{\varepsilon^{2}} \int_{0}^{t} w^{(n)}(\sigma) d \sigma, \\
w^{(n+1)}(t) & =v_{0}+\frac{1}{\varepsilon} \int_{0}^{t} G^{*}\left(\sigma, w^{(n)}(\cdot), z^{(n)}(\sigma), w^{(n)}(\sigma)\right) d \sigma .
\end{aligned}
$$

For fixed $z, w$ and $t<\eta, G^{*}(t, w(\cdot), z, w)$ is clearly $\mathscr{G}_{E_{i}}$ measurable and hence by (3.28) so are $z^{(1)}(t), u^{(1)}(t)$. It then follows by induction on $n$ from (3.28) that $\left(z^{(n)}(t)\right.$, $\left.w^{(n)}(t)\right)$ and also $z^{*}(t), u^{*}(t)$ are $\mathscr{G}_{E_{i}}$ measurable for all $t<\eta$. This remains valid for $t=\eta$ by continuity and the argument can now be repeated for $\eta \leqq t<2 \eta$ etc. It follows that $\left(z^{*}(t), u^{*}(t)\right)$ is $\mathscr{G}_{E_{i}}$ measurable for all $t$. However, it is also clear that $z^{*}(t), u^{*}(t)$ coincides with $z(t), u(t)$ for all $t \leqq S$, where $S=\inf \left\{v \geqq 0: d\left(z(v), C_{i}\right) \geqq\right.$ $\left.\rho-\frac{3}{2} \tau\right\}$. In particular this holds until the first time $z^{*}$ leaves $D_{i}$ and

$$
Y I\left[R \subset D_{i}\right]=Y^{*} I\left[R^{*} \subset D_{i}\right]
$$

where $Y^{*}$ and $R^{*}$ are defined by replacing $z(\cdot), w(\cdot)$ by $z^{*}(\cdot), w^{*}(\cdot)$ in the definition of $Y$ and $R$. Since the right-hand side of (3.29) is $\mathscr{G}_{E_{i}}$ measurable, this implies (3.26).

Now set

$$
F_{i}=\left\{z: d\left(z, C_{i}\right) \leqq \kappa\right\}
$$

and $\mathscr{B}_{i}=$ the collection of Borel sets of $C_{i} \times \mathbb{R}^{d}$. Then the map from $C_{i} \times \mathbb{R}^{d} \times \Omega$ into $\mathbb{R}$ given by $(\theta, \omega) \rightarrow h_{1}\left(\tau_{\theta} F\right)$ is $\mathscr{B}_{i} \times \mathscr{G}_{F_{i}}$ measurable, because for fixed $\theta=$ $\left(\theta^{\prime}, \theta^{\prime \prime}\right) \in C_{i} \times \mathbb{R}^{d}, \theta^{\prime}+\Delta_{i} \subset F_{i}$ and hence $h_{1}\left(\tau_{\theta} F\right)$ is $\mathscr{G}_{F_{i}}$ measurable in $\omega$. Moreover, for fixed $\omega, \tau_{\theta} F$ is continuous in $\theta$. We now combine this with the fact that for any Borel set $B \subset C_{i} \times \mathbb{R}^{d}, X I_{i} I\left[g_{1} \in B\right] I\left[R \subset D_{i}\right]$ is $\mathscr{G}_{E_{i}}$ measurable (by (3.26)) to conclude that

$$
X I_{i} I\left[R \subset D_{i}\right] h_{1}\left(\tau_{g_{1}} F\right) \text { is } \mathscr{G}_{E_{i} \cup F_{i}} \text { measurable. }
$$


In addition

$$
d\left(E_{i}, F_{i}\right) \geqq d\left(C_{i}, E_{i}\right)-\kappa \geqq \rho-2 \tau-\kappa,
$$

so that

$$
|P(A B)-P(A) P(B)| \leqq P(A) \beta(\rho-2 \tau-\kappa)
$$

whenever $A \in \mathscr{G}_{E_{i}}, B \in \mathscr{G}_{F_{i}}$. Let $Q$ be the probability measure on $\mathscr{G}_{E_{i} \cup F_{i}}$ which is defined by $Q(A B)=P(A) P(B)$ whenever $A \in \mathscr{G}_{E_{i}}, B \in \mathscr{G}_{F_{i}} \cdot Q$ is well defined since $\mathscr{G}_{E_{\imath} \cup F_{l}}$ is generated by such sets $A B$. We can then rephrase (3.31) as

$$
|P(A B)-Q(A B)| \leqq P(A) \beta(\rho-2 \tau-\kappa) .
$$

Now let $Z$ be $\mathscr{G}_{E_{i}}$ measurable and $\Gamma$ be $\mathscr{G}_{E_{i} \cup F_{i}}$ measurable. Following [8], p. 171 we shall show that (3.32) implies

$$
\left|\int Z \Gamma d P-\int Z \Gamma d Q\right| \leqq 2 \beta(\rho-2 \tau-\kappa) \sup |\Gamma| E\{|Z|\} \text {. }
$$

Indeed it suffices to prove (3.33) if $Z$ and $\Gamma$ are of the form

$$
Z=\Sigma z_{k} I_{A_{k}}, \quad \Gamma=\Sigma \gamma_{k, l} I_{A_{k} \cap B_{l}}
$$

with $A_{k} \in \mathscr{G}_{E_{l}}$ and $B_{l} \in \mathscr{G}_{F_{i}}$. But for such $Z$ and $\Gamma$,

$$
\begin{aligned}
& \left|\int Z \Gamma d P-\int Z \Gamma d Q\right| \\
& \quad \leqq \sum_{k}\left|z_{k}\right| P\left(A_{k}\right) \sum_{l}\left|\gamma_{k, l}\right|\left|P\left(B_{l} \mid A_{k}\right)-P\left(B_{l}\right)\right| \\
& \quad \leqq E\{|Z|\} \sup |\Gamma| \max _{k} \sum_{l}\left|P\left(B_{l} \mid A_{k}\right)-P\left(B_{l}\right)\right| \\
& \quad \leqq 2 \beta(\rho-2 \tau-\kappa) \sup |\Gamma| E\{|Z|\} .
\end{aligned}
$$

The last inequality is just (20.27) on p. 171 of [8].

We can apply (3.33) to

$$
Z X I_{i} I\left[R \subset D_{i}\right], \quad \Gamma=I_{i} I\left[R \subset D_{i}\right] h_{1}\left(\tau_{g_{1}} F\right),
$$

for which

$$
\int Z \Gamma d Q=E\left\{X I\left[R \subset D_{i}\right] I_{i} U_{1}\left(g_{1}\right)\right\} .
$$

As a result (recall (3.20))

$$
\begin{aligned}
& \left|E\left\{X I_{i} I\left[R \subset D_{i}\right] h_{1}\left(\tau_{g_{1}} F\right)\right\}-E\left\{X I_{i} I\left[R \subset D_{i}\right] U_{1}\left(g_{1}\right)\right\}\right| \\
& \quad \leqq 2 \beta(\rho-2 \tau-\kappa) A E\left\{|X| I_{i}\right\} .
\end{aligned}
$$

Taking into account (3.25) we obtain after summation over $i$

$$
\left|E\left\{X h_{1}\left(\tau_{g_{1}} F\right)\right\}-E\left\{X U_{1}\left(g_{1}\right)\right\}\right| \leqq 2 A \beta(\rho-2 \tau-\kappa) E\{|X|\} .
$$

Formula (3.21) follows by taking the limit as $\tau \downarrow 0$.

We define

$$
\begin{aligned}
& L(\xi, \sigma)=z(\xi)+\frac{\sigma-\xi}{\varepsilon^{2}} w(\xi), \\
& r(s, \xi, \sigma)=s z(\sigma)+(1-s) L(\xi, \sigma) .
\end{aligned}
$$


Lemma 3. Let

$$
H(t, z, w)=D^{\beta} G(t, z, w) \quad \text { or } \quad H=D^{\beta} F(z, w)
$$

for some $|\beta| \leqq 2$ and $D^{\beta}$ only involving derivatives with respect to $z$ and $w$. Then for each fixed $T, M$ there exists a constant $C_{4}$ such that for all

$$
0 \leqq \sigma \leqq T,\left(\sigma-\varepsilon^{3 / 2}\right)^{+} \leqq \xi \leqq \sigma, 0 \leqq s \leqq 1,0<\varepsilon \leqq 1 .
$$

one has

$$
E\left\{\sup _{|w| \leqq 2 M}|H(\sigma, r(s, \xi, \sigma), w)|^{8}\right\} \leqq C_{4}^{8} .
$$

Proof. We only consider an $H$ of the form

$$
H(t, z, w)=D^{\beta_{1}} \Psi(t, w) D^{\beta_{2}} \Phi(t, z) D^{\beta_{3}} F(z, w)
$$

where $D^{\beta_{1}}$ involves only $w$-derivatives and $D^{\beta_{2}}$ only $z$ derivatives. $D^{\beta} G(t, z, w)$ is a finite sum of such terms, and the same estimates can be used if $H=D^{\beta} F(t, w)$.

We shall also restrict ourselves to

$$
0<\varepsilon \leqq \eta \wedge(4 N)^{-8} \wedge T^{-16 d(r(r-8-4 d))^{-1}}
$$

( $r=16 d+64$ again), since (3.37) is immediate from (II) and (IV) for $\varepsilon$ bounded away from zero (and hence $r(s, \xi, \sigma)$ bounded above, on account of (3.11) and (3.13)).

Let

$$
K^{A}(z, w)= \begin{cases}D^{\beta_{3}} F(z, w) & \text { if }\left|D^{\beta_{3}} F(z, w)\right| \leqq A \\ 0 & \text { if }\left|D^{\beta_{3}} F(z, w)\right|>A,\end{cases}
$$

and

$$
\begin{aligned}
& H^{A}(t, z, w)=D^{\beta_{1}} \Psi(t, w) D^{\beta_{2}} \Phi(t, z) K^{A}(z, w), \\
& \tilde{H}^{A}(t, z, w)=H(t, z, w)-H^{A}(t, z, w) .
\end{aligned}
$$

We begin by estimating the error introduced by replacing $H$ by $H^{A}$ in (3.37). Since $D^{\beta_{1}} \Psi(t, w)$ and $D^{\beta_{2}} \Phi(t, z)$ are uniformly bounded in $t \leqq T, w, z \in \mathbb{R}^{d}$ (cf. (3.10)) and

$$
|r(s, \xi, \sigma)| \leqq \frac{2 M}{\varepsilon^{2}} \sigma+\left|y_{0}\right|(\mathrm{cf} .(3.11) \text { and (3.13)) }
$$

this error is

$$
\begin{aligned}
& E\left\{\sup _{|w| \leqq 2 M} \mid \tilde{H}^{A}\left(\sigma, r(s, \xi, \sigma),\left.w\right|^{8}\right\}\right. \\
& \quad \leqq E\left\{\sup _{|w| \leqq 2 M}\left|D^{\beta_{3}} F(r(s, \xi, \sigma), w)-K^{A}(r(s, \xi, \sigma), w\}\right|^{8}\right\} \\
& \quad \leqq A^{8-r} E\left\{\sup _{|z| \leqq 2 M \varepsilon^{-2} \sigma+\left|y_{0}\right|}\left|D^{\beta_{3}} F(z, w)\right|^{r}\right\} \\
& \\
& \quad \leqq C_{5} A^{8-r \mid \leqq 2 M}\left(\frac{\sigma}{\varepsilon^{2}}+1\right)^{d}(\text { by }(\mathrm{IV}))
\end{aligned}
$$


Next we shall replace $r(s, \xi, \sigma)$ by

$$
\begin{aligned}
x(s, \tau, \xi, \sigma) & =s L(\tau, \sigma)+(1-s) L(\xi \wedge \tau, \sigma) \\
& =s\left[z(\tau)+\frac{\sigma-\tau}{\varepsilon^{2}} w(\tau)\right]+(1-s)\left[z(\xi \wedge \tau)+\frac{\sigma-\xi \wedge \tau}{\varepsilon^{2}} w(\xi \wedge \tau)\right]
\end{aligned}
$$

where $\tau$ will be chosen later in such a way that

$$
(\sigma-\eta)^{+} \leqq \tau \leqq \xi \leqq \sigma \quad \text { or } \quad(\sigma-\eta)^{+} \leqq \tau \leqq \sigma-\varepsilon^{7 / 4} \text {. }
$$

We claim that

$$
P\{|r(s, \xi, \sigma)-x(s, \tau, \xi, \sigma)| \geqq 1\} \leqq C_{6} \varepsilon^{-d-2 r}(\sigma-\tau)^{2 r}
$$

Indeed, by (3.9), (3.11) and (3.13),

$$
\begin{aligned}
|z(\sigma)-L(\tau, \sigma)| & =\left|\frac{1}{\varepsilon^{2}} \int_{\tau}^{\sigma}[w(\lambda)-w(\tau)] d \lambda\right| \\
& =\left|\frac{1}{\varepsilon^{3}} \int_{\tau}^{\sigma} d \rho G(\rho, z(\rho), w(\rho))\right| \\
& \leqq \frac{1}{\varepsilon^{3}}(\sigma-\tau)^{2} \sup _{\substack{|z| \leqq 2 M \varepsilon^{-2} T+\left|y_{0}\right| \\
|w| \leqq 2 M}}|F(z, w)|
\end{aligned}
$$

Therefore, as in (3.15), (3.16)

$$
\begin{aligned}
& P\{|z(\sigma)-L(\tau, \omega)| \geqq 1\} \\
& \leqq P\left\{\sup _{|z| \leqq 2 M \varepsilon^{-1} T+\left|y_{0}\right|}|F(z, w)| \geqq \varepsilon^{3}(\sigma-\tau)^{-2}\right\} \\
& \quad \leqq C_{6} \varepsilon^{-2 d-3 r}(\sigma-\tau)^{2 r} .
\end{aligned}
$$

Similarly, for $\tau \leqq \xi$,

$$
\begin{aligned}
& P\{|L(\xi \wedge \tau, \sigma)-L(\xi, \sigma)| \geqq 1\} \\
& \quad \leqq P\left\{\left|z(\xi)-z(\tau)-\frac{\xi-\tau}{\varepsilon^{2}} w(\tau)\right| \geqq \frac{1}{2}\right\}+P\left\{\frac{\sigma-\xi}{\varepsilon^{2}}|w(\xi)-w(\tau)| \geqq \frac{1}{2}\right\} \\
& \quad \leqq C_{6} \varepsilon^{-2 d-3 r}(\sigma-\tau)^{2 r} .
\end{aligned}
$$

For $\tau>\xi$ the first member of (3.45) vanishes. This proves (3.42), since the left-hand side of (3.42) is bounded by the sum of (3.44) and (3.45). From (3.42) we deduce

$$
\begin{aligned}
& E\left\{\sup _{|w| \leqq 2 M}\left|H^{A}(\sigma, r(s, \xi, \sigma), w)\right|^{8}\right\} \\
& \leqq E\left\{\sup _{y|\leqq 1,| w \mid \leqq 2 M}\left|H^{A}(\sigma, x(s, \tau, \xi, \sigma)+y, w)\right|^{8}\right\} \\
&+A^{8} P\{|r(s, \xi, \sigma)-x(s, \tau, \xi, \sigma)| \geqq 1\} \\
& \leqq C_{7} E\left\{\sup _{|y| \leqq 1}\left|D^{\beta_{2}} \Phi(\sigma, x(s, \tau, \xi, \sigma)+y)\right|^{8}\right. \\
& \sup _{|y| \leqq} \mid K^{A}(x \mid \leqq 2 M \\
&+\left.\left.C_{6} A^{8} \varepsilon^{-2 d-3 r}(\sigma, \tau, \xi, \sigma)+y, w\right)\left.\right|^{8}\right\}
\end{aligned}
$$


Now notice that $x(s, \tau, \xi, \sigma)$ is $\mathscr{F}_{\tau}$-measurable. If we further choose $k$ such that $k \eta \leqq \sigma<(k+1) \eta$, then also $\sup D^{\tau_{2}}|\phi(\sigma, x(s, \tau, \xi, \sigma)+y)|$ is $\mathscr{F}_{\tau}$-measurable because $((k-1) \eta)^{+} \leqq \tau$ by $(3.41)$. We shall now apply Lemma 2 to estimate the first term in the last member of (3.46). We choose $t=\tau$,

$$
\begin{aligned}
& \tilde{X}=\sup _{|y| \leqq 1}\left|D^{\beta_{2}} \phi(\sigma, x(s, t, \xi, \sigma)+y)\right|^{8}, \\
& g_{1}^{\prime}=x(s, \tau, \xi, \sigma), \quad g_{1}^{\prime \prime}=0, \quad h_{1}=\sup _{|y| \leqq 1,|w| \leqq 2 M}\left|K^{A}(y, w)\right|^{8} .
\end{aligned}
$$

To apply (3.21) we need a lower bound for

$$
\min \{|x(s, \tau, \xi, \sigma)-z(u)|: u \leqq \tau\}=\rho^{\prime} \wedge \rho^{\prime \prime},
$$

where

$$
\begin{aligned}
\rho^{\prime} & =\min \{|x(s, \tau, \xi, \sigma)-z(u)|: u \leqq(k-1) \eta\}, \\
\rho^{\prime \prime} & =\min \{|x(s, \tau, \xi, \sigma)-z(u)|:(k-1) \eta \leqq u \leqq \tau\} .
\end{aligned}
$$

By definition of $\phi$,

$$
\rho^{\prime} \geqq \frac{\delta}{\varepsilon^{2}}-1 \text { on the set }\{\tilde{X} \neq 0\},
$$

and we merely have to worry about $\rho^{\prime \prime}$. Again by (3.9)

$$
\begin{gathered}
\varepsilon^{2}\left[z(\xi \wedge \tau)+\frac{\sigma-\xi \wedge \tau}{\varepsilon^{2}} w(\xi \wedge \tau)-z(u)\right] \\
=\int_{u}^{\xi \wedge \tau} w(\lambda) d \lambda+(\sigma-\xi \wedge \tau) w(\xi \wedge \tau) .
\end{gathered}
$$

By (3.41), (3.36) and (3.38), $\xi \wedge \tau \geqq((k-1) \eta)^{+}$so that for $((k-1) \eta)^{+} \leqq u \leqq \xi \wedge \tau$, the inner product of $(3.48)$ with $w_{k-1}=|w((k-1) \eta)|^{-1} w((k-1) \eta)\left(|w(0)|^{-1} w(0)\right.$ if $k=0$ ) is by virtue of (3.12) at least

$$
\int_{u}^{\xi \wedge \tau} \frac{1}{2 N} d \lambda+(\sigma-\xi \wedge \tau) \frac{1}{2 N}=(\sigma-u) \frac{1}{2 N} \geqq(\sigma-\tau) \frac{1}{2 N} .
$$

A fortiori, if $\tau \leqq \xi$

$$
\begin{aligned}
& \quad \inf _{(k-1) \eta \leqq u \leqq \tau}\left|z(\xi \wedge \tau)+\frac{\sigma-\xi \wedge \tau}{\varepsilon^{2}} w(\xi \wedge \tau)-z(u)\right| \\
& \quad \geqq \frac{1}{2 N \varepsilon^{2}}(\sigma-\tau) .
\end{aligned}
$$

The same estimate holds if $(\xi \wedge \tau)$ is replaced by $\tau$. Unfortunately (3.49) does not necessarily hold for $\xi<u$. This can occur for some $(k-1) \eta \leqq \xi<u \leqq \tau$ if $\xi<\tau$. In that case we can only conclude that the inner product of (3.48) with $w_{k-1}$ is no less than

$$
\begin{gathered}
(\sigma-u)\left(w(\xi \wedge \tau), w_{k-1}\right)-\left|\int_{u}^{\xi \wedge \tau}\right| w(\lambda)-w(\xi \wedge \tau)|d \lambda| \\
\geqq(\sigma-u) \frac{1}{2 N}-(\sigma-\xi) \sup _{\xi \leqq \lambda \leqq \tau}|w(\lambda)-w(\xi)| .
\end{gathered}
$$


Consider the event (which belongs to $\mathscr{F}_{\tau}$ )

$$
\left\{\sup _{\xi \leqq \lambda \leqq \tau}|w(\lambda)-w(\xi)| \leqq(\sigma-\xi) \varepsilon^{-9 / 8}\right\}
$$

and denote its indicator function by $J$. Then on the set $\{J \neq 0\}$, i.e., when the event (3.51) occurs, the right-hand side of (3.50) is at least

$$
(\sigma-\tau) \frac{1}{2 N}-(\sigma-\xi)^{2} \varepsilon^{-9 / 8} \geqq(\sigma-\tau) \frac{1}{4 N}
$$

(by (3.36), (3.38), and (3.41)). Together with the above estimates this shows that

$$
\rho^{\prime} \wedge \rho^{\prime \prime} \geqq\left(\frac{\delta}{\varepsilon^{2}}-1\right) \wedge \frac{1}{4 N \varepsilon^{2}}(\sigma-\tau) \text { on the set }\{\tilde{X} J \neq 0) .
$$

We can now apply (3.21) with $X=\tilde{X} J$ and $g_{1}$ and $h_{1}$ as in (3.47). We then obtain

$$
\begin{aligned}
& \mid E\left\{\tilde{X} J \sup _{|y| \leqq 1,|w| \leqq 2 M}\left|K^{A}(x(s, \tau, \xi, \sigma)+y, w)\right|^{8}\right\} \\
& \quad \leqq E\left\{|\tilde{X}|\left[U_{1}(x(s, \tau, \xi, \sigma), 0)+2 A^{8} \beta\left(\frac{\delta}{\varepsilon^{2}}-3\right)+2 A^{8} \beta\left(\frac{\sigma-\tau}{4 N \varepsilon^{2}}-2\right)\right]\right\},
\end{aligned}
$$

where

$$
E\{|\tilde{X}|\} \leqq\left(E\left\{|\tilde{X}|^{2}\right\}\right)^{1 / 2} \leqq \sup \left(E\left\{\left|D^{\beta_{2}} \phi(\sigma, z)\right|^{16}\right\}\right)^{1 / 2} \leqq C_{8}<\infty,
$$

and (by (IV))

$$
\begin{aligned}
& U_{1}(x(s, \tau, \xi, \sigma), 0)=E\left\{\sup _{|y| \leqq 1,|w| \leqq 2 M}\left|K^{A}(z+y, w)\right|^{8}\right\} z=x(s, \tau, \xi, \sigma) \\
& \quad \leqq E\left\{\sup _{|y| \leqq 1,|w| \leqq 2 M}\left|D^{\beta_{3}} F(y, w)\right|^{8}\right\} \leqq C_{9} .
\end{aligned}
$$

Of course, by Schwarz' inequality we also have

$$
\begin{aligned}
& \mid E\left\{\tilde{X}(1-J) \sup _{|y| \leqq 1,|w| \leqq 2 M}\left|K^{A}(x(s, \tau, \xi, \sigma)+y, w)\right|^{8}\right\} \\
& \quad \leqq\left(E\left\{\tilde{X}^{2}\right\}\right)^{1 / 2}(P\{J=0\})^{1 / 2} A^{8} \\
& \quad \leqq C_{8} C_{2}^{1 / 2} A^{8} \varepsilon^{4} \quad \text { (by (3.14) and (3.51)). }
\end{aligned}
$$

Combining all these estimates. we finally obtain that the first term on the righthand side of (3.46) is at most

$$
C_{9}+C_{8} A^{8}\left[\beta\left(\frac{\delta}{\varepsilon^{2}}-3\right)+=\left(\frac{\sigma-\tau}{4 N \varepsilon^{2}}-2\right)+C_{2}^{1 / 2} \varepsilon^{4}\right] .
$$

The left-hand side of (3.37) is therefore bounded by (3.52) plus

$$
C_{5} A^{8-r}\left(\frac{\sigma}{\varepsilon^{2}}+1\right)^{d}+C_{6} A^{8} \varepsilon^{-2 d-3 r}(\sigma-\tau)^{2 r} .
$$

It remains to choose $A$ and $\tau$ so that (3.52) and (3.53) are both bounded. We 
may assume that

$$
\frac{\sigma}{\varepsilon^{2}} \geqq 16 N
$$

since, if (3.54) fails, then by (3.13)

$$
|r(s, \xi, \sigma)| \leqq 2 M \cdot 16 N+\left|y_{0}\right|
$$

and (3.37) is immediate from (IV). If (3.54) holds we choose

$$
A=\left(\frac{\sigma}{\varepsilon^{2}}\right)^{d(r-8)^{-1}}
$$

and

$$
\sigma-\tau=\min \left\{\sigma, \varepsilon^{(2 d+3 r) / 2 r}\left(\frac{\varepsilon^{2}}{\sigma}\right)^{8 d(2 r(r-8))^{-1}}\right\}
$$

Note that the second expression in (3.55) is at most $\varepsilon \leqq \eta$ (by virtue of (3.38) and (3.54)) so that the requirement $\tau \geqq(\sigma-\eta)^{+}$of (3.41) is satisfied by (3.55). If we take into account that $\sigma \leqq T$ we immediately see that under (3.54), (3.52) plus (3.53) is bounded by

$$
C_{10}\left\{1+\left(\frac{\sigma}{\varepsilon^{2}}\right)^{8 d(r-8)^{-1}}\left[\beta\left(\frac{\delta}{\varepsilon^{2}}-3\right)+\beta\left(\frac{\sigma-\tau}{4 N \varepsilon^{2}}-2\right)\right]\right\}
$$

Now observe that

$$
\beta(u)=o\left(u^{-24}\right), u \rightarrow \infty, \text { whence } \beta(u) \leqq C_{11}(u+1)^{-24}
$$

by virtue of $(2.2)$ and the fact that $\beta(\cdot)$ is nonincreasing. Therefore (recall $r=16 d+64)$ also

$$
\left(\frac{\sigma}{\varepsilon^{2}}\right)^{8 d(r-8)^{-1}} \beta\left(\frac{\delta}{\varepsilon^{2}}-3\right) \leqq C_{12} .
$$

If the min in (3.55) equals $\sigma$, then we take $\tau=0$ (which always satisfies (3.41) since $\xi \geqq 0$ ) and the remaining term in (3.56) becomes

$$
C_{10}\left(\frac{\sigma}{\varepsilon^{2}}\right)^{8 d(r-8)^{-1}} \beta\left(\frac{\sigma}{4 N \varepsilon^{2}}-2\right) \leqq C_{13}
$$

as above. If the min in (3.55) equals

$$
\begin{aligned}
\varepsilon^{3 / 2+d /(r-8)} \sigma^{-8 d(2 r(r-8))^{-1}} & \geqq \varepsilon^{3 / 2+d /(r-8)} T^{-8 d(2 r(\zeta-8))^{-1}} \\
& \geqq \varepsilon^{7 / 4},
\end{aligned}
$$

(use (3.38) for the last inequality) then $\sigma-\tau$ is given by the left-hand side of (3.58). In this case,

$$
\left(\frac{\sigma}{\varepsilon^{2}}\right)^{8 d(r-8)^{-1}} \beta\left(\frac{\sigma-\tau}{4 N \varepsilon^{2}}-2\right) \leqq T^{8 d(r-8)^{-1}} \varepsilon^{-16 d(r-8)^{-1}} \beta\left(\frac{1}{4 N \varepsilon^{1 / 4}}-2\right)
$$

which (again by (3.57)) is uniformly bounded in $\varepsilon \in(0,1]$. The proof is complete. 
Corollary 1. For $0 \leqq \tau \leqq \sigma \leqq T, p \leqq 8,|\beta| \leqq 2$,

$$
E\left|D^{\beta} G(\sigma, z(\sigma), w(\sigma))\right|^{p} \leqq C_{4}^{p}
$$

and

$$
\left(E\left\{\left|z(\sigma)-z(\tau)-\frac{\sigma-\tau}{\varepsilon^{2}} w(\tau)\right|^{p}\right\}\right)^{1 / p} \leqq C_{4} \frac{(\sigma-\tau)^{2}}{\varepsilon^{3}} .
$$

Proof. Formula (3.59) is immediate from (3.37) with $s=1, \xi=\sigma$ (so that $r(s, \xi, \sigma)=$ $z(\sigma)$ ), and (3.11) and Jensen's inequality (3.60) now follows from Holder's inequality and

$$
z(\sigma)-z(\tau)-\frac{\sigma-\tau}{\varepsilon^{2}} w(\tau)=\frac{1}{\varepsilon^{3}} \int_{\tau}^{\sigma} d \lambda \int_{\tau}^{\lambda} d \rho G(\rho, z(\rho), w(\rho))
$$

(see (3.9)).

\section{Lemma 4. Let}

$$
\begin{aligned}
& 0 \leqq \sigma_{1}, \sigma_{2} \leqq T, \quad k \eta \leqq \sigma_{1} \leqq \sigma_{2}<(k+1) \eta \\
& 0 \leqq \lambda_{1}, \lambda_{2}, \lambda_{3} \leqq \sigma_{1}, \quad\left(\sigma_{2}-\varepsilon^{3 / 2}\right)^{+} \leqq \xi_{2} \leqq \sigma_{2}, \\
& \left(\sigma_{1}-\varepsilon^{25 / 16}\right)^{+} \leqq \xi_{1}, \xi_{2} \leqq \sigma_{1}
\end{aligned}
$$

(notice $\sigma_{1}$ in both sides of the last inequality) and set

$$
v=\max \left\{k \eta, \lambda_{1}, \lambda_{2}, \lambda_{3}, \xi_{1}, \xi_{2}\right\}
$$

Let $\zeta$ be an $\mathscr{F}_{\lambda_{3}}$ measurable random variable with $E \zeta^{2}<\infty$ and

$$
H_{1}(t, z, w)=D^{\beta_{1}} \Psi(t, w) D^{\beta_{2}} \Phi(t, z) D^{\beta_{3}} F(z, w),
$$

and

$$
H_{2}(t, z, w)=D^{\beta_{4}} \Psi(t, w) D^{\beta_{5}} \Phi(t, z) D^{\beta_{6}} F(z, w),
$$

where all derivatives are with respect to $w$ or $z$. Set

$$
V\left(z_{1}, w_{1}, z_{2}, w_{2}\right)=E\left\{D^{\beta_{3}} F\left(z_{1}, w_{1}\right) D^{\beta_{6}} F\left(z_{2}, w_{2}\right)\right\} .
$$

Then (see (3.34) for $L(\cdot, \cdot)$ )

$$
\begin{aligned}
& \mid E\left\{\zeta H_{1}\left(\sigma_{1}, L\left(\xi_{1}, \sigma_{1}\right), w\left(\lambda_{1}\right)\right) H_{2}\left(\sigma_{2}, L\left(\xi_{2}, \sigma_{2}\right), w\left(\lambda_{2}\right)\right)\right\} \\
& -E\left\{\zeta D^{\beta_{1}} \Psi\left(\sigma_{1}, w\left(\lambda_{1}\right)\right) D^{\beta_{4}} \psi\left(\sigma_{2}, w\left(\lambda_{2}\right)\right) D^{\beta_{2}} \Phi\left(\sigma_{1}, L\left(\xi_{1}, \sigma_{1}\right)\right)\right. \\
& \left.\cdot D^{\beta_{5}} \Phi\left(\sigma_{2}, L\left(\xi_{2}, \sigma_{2}\right)\right) V\left(L\left(\xi_{1}, \sigma_{1}\right), w\left(\lambda_{1}\right), L\left(\xi_{2}, \sigma_{2}\right), w\left(\lambda_{2}\right)\right)\right\} \mid \\
& \quad \leqq C_{14}\left(E\left\{\zeta^{2}\right\}\right)^{1 / 2}\left[\beta^{1 / 3}\left(\frac{\delta}{\varepsilon^{2}}\right)+\beta^{1 / 3}\left(\frac{\sigma_{1}-v}{2 N \varepsilon^{2}}-1\right)+\varepsilon^{2}\right]
\end{aligned}
$$

Also, with

$$
\begin{aligned}
& U\left(z_{1}, w_{1}\right)=E\left\{D^{\beta_{3}} F\left(z_{1}, w_{1}\right)\right\}, \quad \pi=\max \left\{k \eta, \lambda_{1}, \lambda_{3}, \xi_{1}\right) \\
& \mid E\left\{\zeta H_{1}\left(\sigma_{1}, L\left(\xi_{1}, \sigma_{1}\right), w\left(\lambda_{1}\right)\right)\right\} \\
& -E\left\{\zeta D^{\beta_{1}} \Psi\left(\sigma_{1}, w\left(\lambda_{1}\right)\right) D^{\beta_{2}} \Phi\left(\sigma_{1}, L\left(\xi_{1}, \sigma_{1}\right)\right) U\left(L\left(\xi_{1}, \sigma_{1}\right), w\left(\lambda_{1}\right)\right)\right\} \mid
\end{aligned}
$$




$$
\leqq C_{14}\left(E\left\{\zeta^{2}\right\}\right)^{1 / 2}\left[\beta^{1 / 2}\left(\frac{\delta}{\varepsilon^{2}}\right)+\beta^{1 / 2}\left(\frac{\sigma_{1}-\pi}{2 N \varepsilon^{2}}-1\right)+\varepsilon^{2}\right]
$$

$C_{14}$ is independent of $\zeta, \sigma_{1}, \xi_{j}, \lambda_{l}$ and $\varepsilon$.

Proof. We only prove (3.62). Define $K_{i}^{A}, H_{i}^{A}$ and $\widetilde{H}_{i}^{A}$ as (3.39), (3.40) and set

$$
V^{A}\left(z_{1}, w_{1}, z_{2}, w_{2}\right)=E\left\{K_{1}^{A}\left(z_{1}, w_{1}\right) K_{2}^{A}\left(z_{2}, w_{2}\right)\right\} .
$$

We first replace $H_{i}$ by $H_{i}^{A}$ and $V$ by $V^{A}$ in the left-hand side of (3.62). The error introduced by the replacement is at most

$$
\begin{gathered}
E\left\{\left|\zeta \widetilde{H}_{1}^{A}\left(\sigma_{1}, L\left(\xi_{1}, \sigma_{1}\right), w\left(\lambda_{1}\right)\right) H_{2}\left(\sigma_{2}, L\left(\xi_{2}, \sigma_{2}\right), w\left(\lambda_{2}\right)\right)\right|\right\} \\
+E\left\{\left|\zeta H_{1}^{A}\left(\sigma_{1}, L\left(\xi_{1}, \sigma_{1}\right), w\left(\lambda_{1}\right)\right) \tilde{H}_{2}^{A}\left(\sigma_{2}, L\left(\xi_{2}, \sigma_{2}\right), w\left(\lambda_{2}\right)\right)\right|\right\} \\
+C_{1}^{4} E\left\{|\zeta| \mid V\left(L\left(\xi_{1}, \sigma_{1}\right), w\left(\lambda_{1}\right), L\left(\xi_{2}, \sigma_{2}\right), w\left(\lambda_{2}\right)\right)\right. \\
\left.\quad-V^{A}\left(L\left(\xi_{1}, \sigma_{1}\right), w\left(\lambda_{1}\right), L\left(\xi_{2}, \sigma_{2}\right), w\left(\lambda_{2}\right)\right) \mid\right\}
\end{gathered}
$$

We estimate the first term of (3.64); it is easily seen that the same bound applies to the other terms. By using (3.10) and (3.37) twice we see that the first term in (3.64) is at most

$$
\begin{aligned}
& C_{1}^{2} E^{1 / 2}\left\{\zeta^{2}\right\} E^{1 / 4}\left\{\mid H_{2}\left(\sigma_{2}, L\left(\xi_{2}, \sigma_{2}\right),\left.w\left(\lambda_{2}\right)\right|^{4}\right\} .\right. \\
& E^{1 / 4}\left\{\mid D^{\beta_{3}} F\left(L\left(\xi_{1}, \sigma_{1}\right),\left.w\left(\lambda_{1}\right)\right|^{4} I\left[\left|D^{\beta_{3}} F\left(L\left(\xi_{1}, \sigma_{1}\right), w\left(\lambda_{1}\right)\right)\right|>A\right]\right\}\right. \\
& \quad \leqq C_{1}^{2} C_{4} E^{1 / 2}\left\{\zeta^{2}\right\} A^{-1} E^{1 / 4}\left\{\mid D^{\beta_{3}} F\left(L\left(\xi_{1}, \sigma_{1}\right),\left.w\left(\lambda_{1}\right)\right|^{8}\right\}\right. \\
& \quad \leqq C_{1}^{2} C_{4}^{3} A^{-1} E^{1 / 2}\left\{\zeta^{2}\right\} .
\end{aligned}
$$

From here on the proof is very similar to Lemma 3. We shall apply (3.22). We make the following choices:

$$
\begin{aligned}
& \widetilde{D}=\zeta D^{\beta_{1}} \Psi\left(\sigma_{1}, w\left(\lambda_{1}\right)\right) D^{\beta_{4}} \Psi\left(\sigma_{2}, w\left(\lambda_{2}\right)\right) \\
& D^{\beta_{2}} \Phi\left(\sigma_{1}, L\left(\xi_{1}, \sigma_{1}\right)\right) D^{\beta_{5}} \Phi\left(\sigma_{2}, L\left(\xi_{2}, \sigma_{2}\right)\right), \\
& g_{i}=\left(L\left(\xi_{i}, \sigma_{i}\right), w\left(\lambda_{i}\right)\right), \\
& h_{1}=D^{\beta_{3}} F(0,0) I\left[\left|D^{\beta_{3}} F(0,0)\right| \leqq A\right], \quad h_{2}=D^{\beta_{6}} F(0,0) I\left[\left|D^{\beta_{6}} F(0,0)\right| \leqq A\right] .
\end{aligned}
$$

Also we define

$$
\begin{aligned}
\rho_{i}^{\prime} & =\min \left\{\left|L\left(\xi_{i}, \sigma_{i}\right)-z(u)\right|: 0 \leqq u \leqq(k-1) \eta\right\}, \\
\rho_{i}^{\prime \prime} & =\min \left\{\left|L\left(\xi_{i}, \sigma_{i}\right)-z(u)\right|:(k-1) \eta \leqq u \leqq v\right\}, \\
J_{i} & =\text { indicator function of }\left\{\sup _{\xi_{\imath} \leqq \lambda \leqq v}\left|w(\lambda)-w\left(\xi_{i}\right)\right| \leqq\left(v-\xi_{i}\right) \varepsilon^{-9 / 8}\right\} .
\end{aligned}
$$

One easily checks that $\tilde{X}$ is $\mathscr{F}_{v}$-measurable. Indeed $\Psi\left(\sigma, w\left(\lambda_{i}\right)\right)$ depends only on $w\left(\lambda_{i}\right)$ and $w((k-1) \eta)$ and $w(k \eta)$, and for the $\Phi$ factors we already checked the appropriate measurability just before (3.47). As in Lemma 3

$$
\rho_{1}^{\prime} \geqq \frac{\delta}{\varepsilon^{2}} \text { on the set }\left\{D^{\beta_{2}} \Phi\left(\sigma_{1}, L\left(\xi_{1}, \sigma_{1}\right)\right) \neq 0\right\},
$$


and similarly for $\rho_{2}^{\prime}$. Also as in Lemma 3 (cf. (3.48)-(3.50)), on the set $\left\{J_{i} \neq 0\right\}$,

$$
\begin{aligned}
& \varepsilon^{2} \rho_{i}^{\prime \prime} \geqq\left(\sigma_{i}-v\right) \frac{1}{2 N}-\left(v-\xi_{i}\right) \sup _{\xi_{\iota} \leqq \lambda \leqq \nu}\left|w(\lambda)-w\left(\xi_{i}\right)\right| \\
& \geqq f\left(\sigma_{i}-v\right) \frac{1}{2 N}-\left(\sigma_{1}-\xi_{i}\right)^{2} \varepsilon^{-9 / 8} \\
& \quad \geqq\left(\sigma_{i}-v\right) \frac{1}{2 N}-\varepsilon^{2} \quad(\text { by }(3.61)) .
\end{aligned}
$$

Thus

$$
\begin{aligned}
& \min _{i=1,2 \quad \min }\left\{\left|L\left(\xi_{i}, \sigma_{i}\right)-z(u)\right|\right\} \\
& \quad=\rho_{1}^{\prime} \wedge \rho_{2}^{\prime} \wedge \rho_{1}^{\prime \prime} \wedge \rho_{2}^{\prime \prime} \geqq \frac{\delta}{\varepsilon^{2}} \wedge\left(\frac{1}{2 N \varepsilon^{2}}\left(\sigma_{1}-v\right)-1\right)
\end{aligned}
$$

on the set $\{X \neq 0\}$, where

$$
X=\tilde{X} J_{1} J_{2} .
$$

Moreover, by (3.14)

$$
P\left\{J_{i}=0\right\} \leqq C_{2} \varepsilon^{8}, \quad i=1,2 .
$$

Now the left-hand side of (3.62) with $H_{i}$ and $V$ replaced by $H_{i}^{A}$, respectively, $V^{A}$, equals

$$
\begin{aligned}
&\left|E\left\{\tilde{X} h_{1}\left(\tau_{g_{1}} F\right) h_{2}\left(\tau_{g_{2}} F\right)\right\}-E\left\{\tilde{X} V^{A}\left(g_{1}, g_{2}\right)\right\}\right| \\
& \leqq\left|E\left\{\tilde{X} h_{1}\left(\tau_{g_{1}} F\right) h_{2}\left(\tau_{g_{2}} F\right)\right\}-E\left\{X V^{A}\left(g_{1}, g_{2}\right)\right\}\right| \\
&+E\left\{|\tilde{X}|\left[\left(1-J_{1}\right)+\left(1-J_{2}\right)\right]\left|h_{1}\left(\tau_{g_{1}} F\right) h_{2}\left(\tau_{g_{2}} F\right)\right|\right\} \\
&+E\left\{|\tilde{X}|\left[\left(1-J_{1}\right)+\left(1-J_{2}\right)\right]\left|V^{A}\left(g_{1}, g_{2}\right)\right|\right\}
\end{aligned}
$$

The first term on the right-hand side of (3.67) is at most

$$
\begin{aligned}
& 2 A^{2} E\{|X|\}\left[\beta\left(\frac{\delta}{\varepsilon^{2}}\right)+\beta\left(\frac{\sigma_{1}-v}{2 N \varepsilon^{2}}-1\right)\right] \\
& \leqq 2 C_{1}^{4} A^{2} E^{1 / 2}\left\{\zeta^{2}\right\}\left[\beta\left(\frac{\delta}{\varepsilon^{2}}\right)+\beta\left(\frac{\sigma_{1}-v}{2 N \varepsilon^{2}}-1\right)\right]
\end{aligned}
$$

on account of (3.22), (3.66) and (3.10).

As for the second term in the right-hand side of (3.67) notice that

$$
\begin{aligned}
& E\left\{|\tilde{X}|\left(1-J_{i}\right)\left|h_{1}\left(\tau_{g_{1}} F\right) h_{2}\left(\tau_{g_{2}} F\right)\right|\right. \\
& \quad=E\left\{|\zeta|\left(1-J_{i}\right)\left|H_{1}^{A}\left(\sigma_{1}, L\left(\xi_{1}, \sigma_{1}\right), w\left(\lambda_{1}\right)\right) H_{2}^{A}\left(\sigma_{2}, L\left(\xi_{2}, \sigma_{2}\right), w\left(\lambda_{2}\right)\right)\right|\right\} . \\
& \quad \leqq E^{1 / 2}\left\{|\zeta|^{2}\right\} E^{1 / 4}\left\{\left(1-J_{i}\right)^{4}\right\} \\
& \quad \cdot\left(E\left\{\left|H_{1}\left(\sigma_{1}, L\left(\xi_{1}, \sigma_{1}\right), w\left(\lambda_{1}\right)\right)\right|^{8}\right\} E\left\{\left|H_{2}\left(\sigma_{2}, L\left(\xi_{2}, \sigma_{2}\right), w\left(\lambda_{2}\right)\right)\right|^{8}\right\}\right)^{1 / 8}
\end{aligned}
$$

By (3.37),

$$
E^{1 / 8}\left\{\left|H_{i}\left(\sigma_{i}, L\left(\xi_{i}, \sigma_{i}\right), w\left(\lambda_{i}\right)\right)\right|^{8}\right\} \leqq C_{4}
$$


so that $(3.68)$ is at most

$$
\begin{gathered}
C_{4}^{2} E^{1 / 2}\left\{\left(\left.\zeta\right|^{2}\right\}\left(P\left\{J_{i}=0\right\}\right)^{1 / 4}\right. \\
\leqq C_{4}^{2} C_{2}^{1 / 4} E^{1 / 2}\left\{|\zeta|^{2}\right\} \varepsilon^{2} .
\end{gathered}
$$

By (IV), $\left|V^{A}\left(z_{1}, w_{1}, z_{2}, w_{2}\right)\right| \leqq C_{0}$ and hence by (3.10) the third term on the righthand side of (3.67) is bounded by

$$
2 C_{0} C_{1}^{4} E^{1 / 2}\left\{\zeta^{2}\right\} C_{2}^{1 / 2} \varepsilon^{4}
$$

Collecting all contribution we find that the left-hand side of (3.62) is bounded by

$$
C_{15} E^{1 / 2}\left\{\zeta^{2}\right\}\left[A^{-1}+A^{2}\left\{\beta\left(\frac{\delta}{\varepsilon^{2}}\right)+\beta\left(\frac{\sigma_{1}-v}{2 N \varepsilon^{2}}-1\right)\right\}+\varepsilon^{2}\right] \text {. }
$$

Formula (3.62) now follows if we take

$$
A=\left\{\beta\left(\frac{\delta}{\varepsilon^{2}}\right)+\beta\left(\frac{\sigma_{1}-v}{2 N \varepsilon^{2}}-1\right)\right\}^{-1 / 3} .
$$

Remark 4. For the sequel we set

$$
\gamma(u)=\left\{\beta\left(\frac{u}{2 N}-1\right)\right\}^{1 / 3}
$$

We then have (see (3.57))

$$
\gamma(u)=o\left(u^{-8}\right), \gamma(u) \leqq C_{11}^{1 / 3}\left(\frac{u}{2 N}+1\right)^{-8} \text { and } \int_{0}^{\infty} u \gamma(u) d u<\infty,
$$

and from (3.61) it follows that we may replace the right-hand sides of (3.62) and (3.63) by

$$
C_{16} E^{1 / 2}\left\{\zeta^{2}\right\}\left\{\varepsilon^{2}+\gamma\left(\frac{\sigma_{1}-\nu}{\varepsilon^{2}}\right)\right\} \text { respectively } C_{16} E^{1 / 2}\left\{\zeta^{2}\right\}\left\{\varepsilon^{2}+\gamma\left(\frac{\sigma_{1}-\pi}{\varepsilon^{2}}\right)\right\} .
$$

Lemma 5. If the hypotheses of Lemma 4 hold, as well as $\xi_{1}=\xi_{2}=\xi$ and $E\{F(z, w)\} \equiv 0$, then the left-hand side of (3.62) is also bounded by

$$
C_{17} E^{1 / 2}\left\{\zeta^{2}\right\}\left\{\varepsilon^{2}+\gamma\left(\frac{\sigma_{1}-v}{\varepsilon^{2}}\right)\right\}^{1 / 2}\left\{\varepsilon^{2}+\gamma\left(\frac{\sigma_{2}-\sigma_{1}}{\varepsilon^{2}}\right)\right\}^{1 / 2}
$$

Proof. We shall show that the left-hand side of (3.62) is also bounded by

$$
C_{18} E^{1 / 2}\left\{\zeta^{2}\right\}\left\{\varepsilon^{2}+\gamma\left(\frac{\sigma_{2}-\sigma_{1}}{\varepsilon^{2}}\right)\right\} .
$$

Formula (3.73) then follows by taking the geometric mean of the bounds (3.71) and (3.74). Formula (3.74) is proved in the same way as (3.63). We choose $\tilde{X}, g_{i}, h_{i}$ and $J_{i}$ as in Lemma 4 (see (3.65) and the preceding lines) and again we take $X=$ 
$\bar{X} J_{1} J_{2}$. This time we begin the estimate of (3.62) with the term

$$
\begin{aligned}
& \mid E\left\{\zeta H _ { 1 } ^ { A } \left(\sigma_{1} L\left(\xi_{1}, \sigma_{1}\right), w\left(\lambda_{1}\right)\left(H_{2}^{A}\left(\sigma_{2}, L\left(\xi_{2}, \sigma_{2}\right), w\left(\lambda_{2}\right)\right)\right\} \mid\right.\right. \\
& \quad=\left|E\left\{\tilde{X} h_{1}\left(\tau_{g_{1}} F\right) h_{2}\left(\tau_{g_{2}} F\right)\right\}\right| \\
& \quad \leqq \\
& \quad\left|E\left\{X h_{1}\left(\tau_{g_{1}} F\right) h_{2}\left(\tau_{g_{2}} F\right)\right\}\right| \\
& \quad+E\left\{|\tilde{X}|\left[\left(1-J_{1}\right)+\left(1+J_{2}\right)\right]\left|h_{1}\left(\tau_{g_{1}} F\right) h_{2}\left(\tau_{g_{2}} F\right)\right|\right\}
\end{aligned}
$$

In the present situation

$$
\begin{aligned}
U_{2}(\theta) & =E\left\{h_{2}\left(\tau_{\theta} F\right)\right\}=E\left\{D^{\beta_{6}} F(\theta) I\left[\left|D^{\beta_{6}} F(0)\right| \leqq A\right]\right\} \\
& =E\left\{D^{\beta_{6}} F(\theta) I\left[\left|D^{\beta_{6}} F(\theta)\right|>A\right]\right\},
\end{aligned}
$$

because (2.1) implies

$$
E\left\{D^{\beta_{6}} F(0)\right\}=0 .
$$

Thus

$$
\left|U_{2}(\theta)\right| \leqq C_{0} A^{-1} \text {. }
$$

In addition, by (3.12)

$$
\begin{aligned}
\left|g_{2}^{\prime}-g_{1}^{\prime}\right| & =\left|L\left(\xi, \sigma_{2}\right)-L\left(\xi, \sigma_{1}\right)\right|=\frac{1}{\varepsilon^{2}}\left|\sigma_{2}-\sigma_{1}\right||w(\xi)| \\
& \geqq \frac{1}{2 N \varepsilon^{2}}\left(\sigma_{2}-\sigma_{1}\right) .
\end{aligned}
$$

Therefore, by (3.24) and the lower bound for $\rho_{2}^{\prime} \wedge \rho_{2}^{\prime \prime}$ in Lemma 4,

$$
\begin{aligned}
& \left|E\left\{X h_{1}\left(\tau_{g_{1}} F\right) h_{2}\left(\tau_{g_{2}} F\right)\right\}\right| \\
& \quad \leqq\left|E\left\{X h_{1}\left(\tau_{g_{1}} F\right) U_{2}\left(g_{2}\right)\right\}\right|+2 A^{2} E\{|X|\}\left\{\beta\left(\frac{\delta}{\varepsilon^{2}}\right)+\beta\left(\frac{\sigma_{2}-\sigma_{1}}{2 N \varepsilon^{2}}-1\right)\right\} \\
& \quad \leqq C_{19} E^{1 / 2}\left\{\zeta^{2}\right\}\left[A^{-1}+A^{2}\left\{\beta\left(\frac{\delta}{\varepsilon^{2}}\right)+\beta\left(\frac{\sigma_{2}-\sigma_{1}}{2 N \varepsilon^{2}}-1\right)\right\}\right]
\end{aligned}
$$

The second term on the right-hand side of (3.75) is at most

$$
2 C_{4}^{2} C_{2}^{1 / 4} E^{1 / 2}\left\{\zeta^{2}\right\} \varepsilon^{2},
$$

as in (3.68), (3.69). We combine this with the estimate for (3.64), and as before we take

$$
A=\left\{\beta\left(\frac{\delta}{\varepsilon^{2}}\right)+\beta\left(\frac{\sigma_{2}-\sigma_{1}}{2 N \varepsilon^{2}}-1\right)\right\}^{-1 / 3} .
$$

There results

$$
\begin{aligned}
& \left|E\left\{\zeta H_{1}\left(\sigma_{1}, L\left(\xi, \sigma_{1}\right), w\left(\lambda_{1}\right)\right) H_{2}\left(\sigma_{2}, L\left(\xi, \sigma_{2}\right), u\left(\lambda_{2}\right)\right)\right\}\right| \\
& \quad \leqq C_{20} E^{1 / 2}\left\{\zeta^{2}\right\}\left\{\varepsilon^{2}+\gamma\left(\frac{\sigma_{2}-\sigma_{1}}{\varepsilon^{2}}\right)\right\} .
\end{aligned}
$$


Lastly, it follows from (3.76), (IV) and Theorem 17.2.3 of [7] or Lemma 20.1 of [8] that

$$
\begin{aligned}
\left|V\left(z_{1}, w_{1}, z_{2}, w_{2}\right)\right| & =\mid E\left\{D^{\beta_{3}} F\left(z_{1}, w_{1}\right) D^{\beta_{6}} F\left(z_{2}, w_{2}\right)\right\} \\
& \leqq 2 C_{0}^{2} \beta^{1 / 2}\left(\left|z_{1}-z_{2}\right|\right) .
\end{aligned}
$$

For $z_{i}=g_{i}^{\prime}=L\left(\xi, \sigma_{i}\right)$ we obtain from this and (3.77)

$$
\begin{aligned}
& \left|E\left\{\zeta V\left(L\left(\xi, \sigma_{1}\right), w\left(\lambda_{1}\right), L\left(\xi, \sigma_{2}\right), w\left(\lambda_{2}\right)\right)\right\}\right| \\
& \quad \leqq 2 C_{0}^{2} \beta^{1 / 2}\left(\left|z_{1}-z_{2}\right|\right) E\{|\zeta|\} \\
& \quad \leqq 2 C_{0}^{2} E^{1 / 2}\left\{\zeta^{2}\right\}\left\{\varepsilon^{2}+\gamma\left(\frac{\sigma_{2}-\sigma_{1}}{\varepsilon^{2}}\right)\right\} .
\end{aligned}
$$

This, together with (3.78) implies (3.74).

\section{Step (iii)}

In this step we prove that the family of measures $\left\{R^{\varepsilon}(\cdot): 0<\varepsilon \leqq 1\right\}=$ $\left\{R^{\varepsilon}(\cdot ; M, N, \eta, \delta): 0<\varepsilon \leqq 1\right\}$ introduced in step (i) is tight in $D$.

As pointed out in step (iii) of [6] it certainly suffices for this to prove

$$
E\left\{\zeta|w(u)-w(t)|^{2}\right\} \leqq C_{21}(u-t) E^{1 / 4}\left\{\zeta^{4}\right\}
$$

For $\zeta \mathscr{F}_{t}$ measurable and $0 \leqq t \leqq u \leqq T$. To prove (3.81) it suffices to restrict oneself to

$$
k \eta \leqq t \leqq u<(k+1) \eta
$$

for some $k \leqq T / \eta$. For once (3.81) has been proved for such $t, u$, then it also holds by continuity for $k \eta \leqq t \leqq u \leqq(k+1) \eta$, whereas for $(k+1) \eta \leqq u<(k+2) \eta$, (3.81) (with $k$ replaced by $(k+1)$ ) gives

$$
E\left(\zeta|w(u)-w(k+1)|^{2}\right\} \leqq C_{21}(u-(k+1) \eta) E^{1 / 4}\left\{\zeta^{4}\right\}
$$

so that (3.81) under (3.82) for each $k \leqq T / \eta$ implies that also for $k \eta \leqq t \leqq(k+1) \eta \leqq$ $u<(k+2) \eta$,

$$
\begin{aligned}
& E\left\{\zeta|w(u)-w(t)|^{2}\right\} \leqq 2 E\left\{\zeta|w((k+1) \eta)-w(t)|^{2}\right\} \\
& +2 E\left\{\zeta|w(u)-w((k+1) \eta)|^{2}\right\} \leqq 2 C_{21}(u-t) E^{1 / 4}\left\{\zeta^{4}\right\} .
\end{aligned}
$$

For $t \leqq(k+1) \eta$ and $u \geqq(k+2) \eta$ we would have $u-t \geqq \eta$ and then (3.81) is trivial with $C_{21}=4 M \eta^{-1}$ since $|w(u)-w(t)| \leqq 4 M$ by (3.11).

The proof of (3.81) under (3.82) is very similar to step (iii) of [6]. We shall use the summation convention and write

$$
D_{2 j} G(\sigma, z(\sigma), w(\sigma)) \text { for } \frac{\partial}{\partial z_{j}} G(\sigma, z(\sigma), u(\sigma))
$$

and similarly for $D_{3 j}$. We take

$$
\xi=\xi(\sigma)=\max \left\{t, \sigma-\varepsilon^{7 / 4}\right\} .
$$


Then, by (3.9)

$$
\begin{aligned}
|w(u)-w(t)|^{2}= & \frac{2}{\varepsilon} \int_{t}^{u} G_{i}(\sigma, z(\sigma), w(\sigma))\left(w_{i}(\sigma)-w_{i}(t)\right) d \sigma \\
= & \frac{2}{\varepsilon} \int_{t}^{u} G_{i}(\sigma, z(\sigma), w(\xi))\left(w_{i}(\xi)-w_{i}(t)\right) d \sigma \\
& +\frac{2}{\varepsilon^{2}} \int_{t}^{u} d \sigma \int_{\xi}^{\sigma}\left[D_{3 j} G_{i}(\sigma, z(\sigma), w(\lambda))\left(w_{i}(\lambda)-w_{i}(t)\right)\right. \\
& \left.+\delta_{i, j} G_{i}(\sigma, z(\sigma), w(\lambda))\right] G_{j}(\lambda, z(\lambda), w(\lambda)) d \lambda \\
= & I_{1}+I_{2} .
\end{aligned}
$$

In $I_{1}$ we replace $z(\sigma)$ by $L(\xi, \sigma)$. More precisely, we write (in the notation of (3.34), (3.35))

$$
\begin{aligned}
I_{1}= & \frac{2}{\varepsilon} \int_{t}^{u} G_{i}(\sigma, L(\xi, \sigma), w(\xi))\left(w_{i}(\xi)-w_{i}(t)\right) d \sigma \\
& +\frac{2}{\varepsilon} \int_{t}^{u} d \sigma \int_{0}^{1} D_{2 j} G_{i}(\sigma, r(s, \xi, \sigma), w(\xi))\left(w_{i}(\xi)-w_{i}(t)\right) \\
& \cdot\left[z_{j}(\sigma)-L_{j}(\xi, \sigma)\right] d s \\
= & J_{1}+J_{2} .
\end{aligned}
$$

By (3.9) again

$$
\begin{aligned}
J_{1}= & \frac{2}{\varepsilon^{2}} \int_{t}^{u} d \sigma \int_{t}^{\xi} d \lambda\left[D_{3 j} G_{i}(\sigma, L(\xi, \sigma), w(\lambda))\left(w_{i}(\lambda)-w_{i}(t)\right)\right. \\
& \left.+\delta_{i j} G_{i}(\sigma, L(\xi, \sigma), w(\lambda))\right] G_{j}(\lambda, z(\lambda), w(\lambda)) .
\end{aligned}
$$

Moreover, by (3.63) and Remark 4 with $\zeta$ replaced by

$$
\zeta\left(w_{i}(\lambda)-w_{i}(t)\right) G_{j}(\lambda, z(\lambda), w(\lambda))
$$

we have for fixed $i$ and $j$ and $\lambda \leqq \xi$,

$$
\begin{aligned}
& \left|E\left\{\zeta D_{3 j} G_{i}(\sigma, L(\xi, \sigma), w(\lambda))\left(w_{i}(\lambda)-w_{i}(t)\right) G_{j}(\lambda, z(\lambda), w(\lambda))\right\}\right| \\
& \quad \leqq C_{16} E^{1 / 2}\left\{\left|\zeta\left(w_{i}(\lambda)-w_{i}(t)\right) G_{j}(\lambda, z(\lambda), w(\lambda))\right|^{2}\right\} \\
& \quad\left\{\varepsilon^{2}+\gamma\left(\frac{\sigma-\xi}{\varepsilon^{2}}\right)\right\} \leqq C_{16} C_{4} E^{1 / 4}\left\{\zeta^{4}\right\}\left\{\varepsilon^{2}+\gamma\left(\frac{\sigma-\xi}{\varepsilon^{2}}\right)\right\}
\end{aligned}
$$

(recall that by (3.82) and (3.83) $\xi \geqq t \geqq k \eta$ and that (3.76) holds; in the last step we also used (3.59)). The other term in $J_{1}$ is handled similarly, so that

$$
\begin{aligned}
\left|E\left\{\zeta J_{1}\right\}\right| & \leqq \frac{C_{22}}{\varepsilon^{2}} E^{1 / 4}\left\{\zeta^{4}\right\} \int_{t}^{u} d \sigma \int_{t}^{\xi} d \lambda\left\{\varepsilon^{2}+\gamma\left(\frac{\sigma-\xi}{\varepsilon^{2}}\right)\right\} \\
& \leqq C_{22} E^{1 / 4}\left\{\zeta^{4}\right\}(u-t)\left\{T+\int_{0}^{\infty} \gamma(t) d t+T \varepsilon^{-2} \gamma\left(\varepsilon^{-1 / 4}\right)\right\} \\
& \leqq C_{23} E^{1 / 4}\left\{\zeta^{4}\right\}(u-t) \quad \text { (see (3.83) and (3.71)). }
\end{aligned}
$$

Next we replace $r(s, \xi, \sigma)$ in $J_{2}$ also by $L(\xi, \sigma)$. Note that for $0 \leqq v \leqq 1$, $v r(s, \xi, \sigma)+(1-v) L(\xi, \sigma)=r(v s, \xi, \sigma)$ 
(see (3.34), (3.35)), so that

$$
\begin{aligned}
J_{2}= & \frac{2}{\varepsilon} \int_{t}^{u} d \sigma \int_{0}^{1} D_{2 j} G_{i}(\sigma, L(\xi, \sigma), w(\xi))\left(w_{i}(\xi)-w_{i}(t)\right) \\
& \cdot\left[z_{j}(\sigma)-L_{j}(\xi, \sigma)\right] d s \\
& +\frac{2}{\varepsilon} \int_{t}^{u} d \sigma \int_{0}^{1} d s \int_{0}^{1} d v D_{2 l} D_{2 j} G_{i}(\sigma, r(v s, \xi, \sigma), w(\xi))\left(w_{i}(\xi)-w_{i}(t)\right) \\
& \cdot\left[z_{j}(\sigma)-L_{j}(\xi, \sigma)\right] s\left[z_{l}(\sigma)-L_{l}(\xi, \sigma)\right] \\
= & K_{1}+K_{2} .
\end{aligned}
$$

As in (3.43)

$$
\begin{aligned}
\zeta K_{1}= & \frac{2}{\varepsilon^{4}} \int_{t}^{u} d \sigma D_{2 j} G_{i}(\sigma, L(\xi, \sigma), w(\xi))\left(w_{i}(\xi)-w_{i}(t)\right) \\
& \cdot \int_{\xi}^{\sigma} d \lambda \int_{\xi}^{\lambda} d \rho G_{j}(\rho, z(\rho), w(\rho)) \zeta .
\end{aligned}
$$

Again by (3.63) and Remark 4, this time with $\zeta$ replaced by

$$
\zeta G_{j}(\rho, z(\rho), w(\rho))\left(w_{i}(\xi)-w_{i}(t)\right)
$$

we find

$$
\begin{aligned}
\left|E\left\{\zeta K_{1}\right\}\right| & \leqq \frac{2 C_{16}}{\varepsilon^{4}} E^{1 / 4}\left\{\zeta^{4}\right\} \int_{t}^{u} d \sigma \int_{\xi}^{\sigma} d \lambda \int_{\xi}^{\lambda} d \rho\left\{\varepsilon^{2}+\gamma\left(\frac{\sigma-\rho}{p 2}\right)\right\} \\
& =2 C_{16} E^{1 / 4}\left\{\zeta^{4}\right\} \int_{t}^{u} d \sigma \int_{\xi}^{\sigma} d \rho \varepsilon^{-4}(\sigma-\rho)\left\{\varepsilon^{2}+\gamma\left(\frac{\sigma-\rho}{\varepsilon^{2}}\right)\right\} \\
& \leqq C_{23} E^{1 / 4}\left\{\zeta^{4}\right\}(u-t) \quad \text { (again use (3.83) and (3.71)). }
\end{aligned}
$$

Finally $\zeta K_{2}$ can be estimated directly by (3.11), Holder's inequality, (3.60), Lemma 3 and (3.83),

$$
\begin{aligned}
& \left|E\left\{\zeta K_{2}\right\}\right| \leqq \frac{4 M}{\varepsilon} E^{1 / 4}\left\{\zeta^{4}\right\} \int_{t}^{u} d \sigma \int_{0}^{1} d s \int_{0}^{1} d v E^{1 / 2}\left\{|z(\sigma)-L(\xi, \sigma)|^{4}\right\} \\
& \cdot E^{1 / 4}\left\{\left|D_{2 l} D_{2 j} G_{i}(\sigma, r(v s, \xi, \sigma), w(\xi))\right|^{4}\right\} \\
& \quad \leqq 4 M C_{4} E^{1 / 4}\left\{\zeta^{4}\right\} \int_{t}^{u} d \sigma \frac{(\sigma-\xi)^{4}}{\varepsilon^{7}} \leqq C_{23} E^{1 / 4}\left\{\zeta^{4}\right\}(u-t) .
\end{aligned}
$$

Thus all pieces of $\zeta I_{1}$ are of the order claimed in (3.81). $\zeta I_{2}$ is handled in the same way. For brevity denote the expression in square brackets in $I_{2}$ by $K_{j}(\sigma, z(\sigma), w(\lambda))$. Then

$$
\begin{aligned}
\zeta I_{2}= & \frac{2}{\varepsilon^{2}} \int_{t}^{u} d \sigma \int_{\xi}^{\sigma} d \lambda \zeta K_{j}(\sigma, L(\xi, \sigma), w(\lambda)) G_{j}(\lambda, z(\lambda), w(\lambda)) \\
& +\frac{2}{\varepsilon^{2}} \int_{t}^{u} d \sigma \int_{\xi}^{\sigma} d \lambda \int_{0}^{1} d s \zeta D_{2, l} K_{j}(\sigma, r(s, \xi, \sigma), w(\lambda)) \\
& \cdot G_{j}(\lambda, z(\lambda), w(\lambda))\left[z_{l}(\sigma)-L_{l}(\xi, \sigma)\right] .
\end{aligned}
$$


The first part of $\zeta I_{2}$ can again be estimated by (3.63) (compare $\zeta J_{1}$ ) whereas the second integral is at most

$$
\begin{gathered}
C_{24} \frac{1}{\varepsilon^{2}} E^{1 / 4}\left\{\zeta^{4}\right\} \int_{0}^{u} d \sigma \int_{\xi}^{\sigma} d \lambda \frac{(\sigma-\xi)^{2}}{\varepsilon^{3}} \\
\leqq C_{24} \varepsilon^{1 / 4} E^{1 / 4}\left\{\zeta^{4}\right\}(u-t),
\end{gathered}
$$

on account of (3.37), (3.60) and (3.83).

This completes the proof of (3.61) and hence the tightness of $\left\{R^{\varepsilon}: 0<\varepsilon \leqq 1\right\}$.

Step (iv)

It follows from step (iii) that any sequence $\varepsilon_{n} \downarrow 0$ can be refined such that

$$
R^{\varepsilon_{n}}\left(\cdot ; M, N, \frac{1}{p}, \frac{1}{q}\right) \Rightarrow R\left(\cdot ; M, N, \frac{1}{p}, \frac{1}{q}\right) \text { on } D
$$

as $n \rightarrow \infty$ for all quadruples of integers $M, N, p, q \geqq 1$, with $R(\cdot ; M, N, 1 / p, 1 / q)$ some probability measure on $D$. We denote by $X(t)$ the $t$-coordinate function on $D$, and the corresponding $\sigma$-fields of subsets of $D$ are given by

$$
\mathscr{M}_{v}^{u}=\sigma \text {-field generated by }\left\{X_{t}: u \leqq t \leqq v\right\} \text {. }
$$

The first step towards proving the convergence of $Q^{\varepsilon}$ will be to show that

$$
f\left(X_{t}\right)-\int_{0}^{t}\left(L^{M, N, p, q} f\right)(\sigma, X) d \sigma
$$

is an $\left(R(\cdot ; M, N, 1 / p, 1 / q), \mathscr{M}_{t}^{0}\right)$ martingale for any $C^{\infty}$ function $f: \mathbb{R}^{d} \rightarrow \mathbb{R}$ with compact support and the following definition of $L^{M, N, p, q}$ : Take $\eta=1 / p$ and $\delta=1 / q$ and let

$$
\Psi_{*}(t, X)=\Psi_{*}^{M, N, p}(t, X)=\Psi(t, X, X(t))
$$

where $\Psi$ is as in (3.3) for given $M, N$ and $\eta=1 / p$. Also take

$$
\Phi_{*}(t, X)=\Phi_{*}^{p, q}(t, X)=\phi_{k}\left(X, \int_{0}^{t} X(u) d u\right)
$$

for $k / p \leqq t<(k+1) / p$, where $\phi_{k}$ is as in (3.5) for $\eta=1 / p, \delta=1 / q$. One easily checks (compare the observation just before Lemma 1)

$$
\Phi_{*}(t, w(\cdot))=\Phi(t, w(\cdot), z(t)) .
$$

Further we define for $v \in \mathbb{R}^{d}$ the following coefficients:

$$
\begin{aligned}
& a_{i j}(v)=\int_{-\infty}^{+\infty} E\left\{F_{i}(0, v) F_{j}(t v, v)\right\} d t, \\
& \begin{aligned}
c_{i j}(\sigma, X, v) & =c_{i j}^{M, N, p}(\sigma, X, v) \\
& =\Psi(\sigma, X, v) \int_{0}^{\infty} t E\left\{F_{j}(0, v) \frac{\partial}{\partial x_{j}} F_{i}(t v, v)\right\} d t
\end{aligned}
\end{aligned}
$$




$$
\begin{aligned}
& \left(\text { of course } \frac{\partial}{\partial x_{j}} F(t v, v)=\left[\frac{\partial}{\partial x_{j}} F(x, v)\right]_{x=t v} \text { here }\right) \text {, } \\
& d_{i j}(\sigma, X, v)=d_{i j}^{M, N, p}(\sigma, X, v) \\
& =\left(\frac{\partial}{\partial v_{j}} \Psi(\sigma, X, v)\right) \int_{0}^{\infty} E\left\{F_{j}(0, v) F_{i}(t v, v)\right\} d t, \\
& e_{i j}(\sigma, X, v)=e_{i j}^{M, N, p}(\sigma, X, v) \\
& =\Psi(\sigma, X, v) \int_{0}^{\infty} E\left\{F_{j}(0, v) \frac{\partial}{\partial v_{j}} F_{i}(t v, v)\right\} d t, \\
& b_{i}(\sigma, X, v)=b_{i}^{M, N, p}(\sigma, X, v) \\
& =\sum_{j=1}^{d}\left\{c_{i j}(\sigma, X, v)+d_{i j}(\sigma, X, v)+e_{i j}(\sigma, X, v)\right\} .
\end{aligned}
$$

Finally,

$$
\begin{aligned}
\left(L^{M, N, p, q} f\right)(\sigma, X)= & \Psi_{*}(\sigma, X) \Phi_{*}^{2}(\sigma, X)\left\{\frac{1}{2} \sum_{i, j} \Psi_{*}(\sigma, X) a_{i j}(X(\sigma)) \frac{\partial^{2}}{\partial v_{i} \partial v_{j}} f(X(\sigma))\right. \\
& \left.+\sum_{i} b_{i}^{M, N, p}(\sigma, X, X(\sigma)) \frac{\partial}{\partial v_{i}} f(X(\sigma))\right\} .
\end{aligned}
$$

As shown in step (v) of [6], to prove that (3.85) is an $\left(R(\cdot ; M, N, 1 / p, 1 / q), \mathscr{M}_{t}^{0}\right)$ martingale it suffices to show that

$$
\begin{aligned}
& \lim _{n \rightarrow \infty} E\left\{\left[f\left(w^{\varepsilon_{n}}(u)\right)-f\left(w^{\varepsilon_{n}}(t)\right)\right] \zeta\left(w^{\varepsilon_{n}}(\cdot)\right)\right\} \\
& =E^{M, N, p, q}\left\{\int_{t}^{u} \zeta(X(\cdot))\left(L^{M, N, p, q} f\right)(\sigma, X) d \sigma\right\}
\end{aligned}
$$

for $t \leqq u$ and $\zeta(X(\cdot))$ a bounded continuous function of $X\left(t_{1}\right), X\left(t_{2}\right), \ldots, X\left(t_{m}\right)$ for any $0 \leqq t_{1}<t_{2}<\ldots<t_{m} \leqq t$. It is clear that it suffices to prove (3.88) for $k \eta \leqq t \leqq u<(k+1) \eta$ for some $k$. Indeed, if it is true for such pairs $t, u$, then by continuity it also holds for $k \eta \leqq t \leqq u \leqq(k+1) \eta$ and the general case can then be obtained by iteration. E.g. if $k \eta \leqq t<(k+1) \eta \leqq u<(k+2) \eta$ we merely have to write the left-hand side of (3.88) as

$$
\begin{aligned}
& \lim E\{[f(w(u))-f(w(k+1) \eta)] \zeta\} \\
& +\lim E\{f(w((k+1) \eta)-f(w(t))] \zeta\}
\end{aligned}
$$

and to apply (3.88) to each of these limits separately.

From now on we assume $k \eta \leqq t \leqq u<(k+1) \eta$. As in [6] we rewrite the left- 
hand side of (3.88) by means of (3.9):

$$
\begin{aligned}
& {[f(w(u))-f(w(t))]} \\
& =\frac{1}{\varepsilon} \int_{t}^{u} \frac{\partial f}{\partial w_{i}}(w(\sigma)) G_{i}(\sigma, z(\sigma), w(\sigma)) d \sigma \\
& =\frac{1}{\varepsilon} \int_{t}^{u} \frac{\partial f}{\partial w_{i}}(w(\xi)) G_{i}(\sigma, z(\sigma), w(\xi)) d \sigma \\
& \quad+\frac{1}{\varepsilon^{2}} \int_{t}^{u} d \sigma \int_{\xi}^{\sigma} d \lambda\left[\frac{\partial^{2} f}{\partial w_{j} \partial w_{i}}(w(\lambda)) G_{i}(\sigma, z(\sigma), w(\lambda))\right. \\
& \left.\quad+\frac{\partial f}{\partial w_{i}}(w(\lambda)) D_{3, j} G_{i}(\sigma, z(\sigma), w(\lambda))\right] G_{j}(\lambda, z(\lambda), w(\lambda)) \\
& =I_{1}+I_{2},
\end{aligned}
$$

where we take

$$
\xi=\xi(\sigma)=\max \left\{t, \sigma-\varepsilon^{15 / 8}\right\}
$$

We shall only analyze $I_{1} . I_{2}$ can be handled in the same way. As in the previous step we start with replacing $z(\sigma)$ by $L(\xi, \sigma)$, i.e., we rewrite $I_{1}$ as

$$
\begin{aligned}
& \frac{1}{\varepsilon} \int_{t}^{u} \frac{\partial f}{\partial w_{i}}(w(\xi)) G_{i}(\sigma, L(\xi, \sigma), w(\xi)) d \sigma \\
& +\frac{1}{\varepsilon} \int_{t}^{u} d \sigma \int_{0}^{1} d s \frac{\partial f}{\partial w_{i}}(w(\xi)) D_{2, j} G_{i}(\sigma, r(s, \xi, \sigma), w(\xi)) \\
& \cdot\left[z_{j}(\sigma)-L_{j}(\xi, \sigma)\right]=J_{1}+J_{2} .
\end{aligned}
$$

By (3.63) and Remark 5 with $\zeta$ replaced by $\zeta\left(\partial f / \partial w_{i}\right)(w(\xi))$,

$$
\begin{aligned}
\left|E\left\{\zeta J_{1}\right\}\right| \leqq & C_{16} \sup \left|\frac{\partial f}{\partial w_{i}}\right| E^{1 / 2}\left\{\zeta^{2}\right\} \\
& \cdot \frac{1}{\varepsilon} \int_{t}^{u}\left\{\varepsilon^{2}+\gamma\left(\frac{\sigma-\xi}{\varepsilon^{2}}\right)\right\} d \sigma,
\end{aligned}
$$

so that $E\left\{\zeta J_{1}\right\} \rightarrow 0$ as $\varepsilon \downarrow 0$ (see (3.71) and (3.89)). As in the analysis of $J_{2}$ in step (iii) we replace $r(s, \xi, \sigma)$ again by $L(\xi, \sigma)$ in $J_{2}$ :

$$
\begin{aligned}
J_{2}= & \frac{1}{\varepsilon} \int_{t}^{u} d \sigma \frac{\partial f}{\partial w_{i}}(w(\xi)) D_{2, j} G_{i}(\sigma, L(\xi, \sigma), w(\xi)) \\
& \cdot\left[z_{j}(\sigma)-L_{j}(\xi, \sigma)\right] \\
& +\frac{1}{\varepsilon_{t}} \int_{t}^{u} d \sigma \int_{0}^{1} d s \int_{0}^{1} d v \frac{\partial f}{\partial w_{i}}(w(\xi)) D_{2, l} D_{2, j} G(\sigma, r(v s, \xi, \sigma), w(\xi)) \\
& \cdot\left[z_{j}(\sigma)-L_{j}(\xi, \sigma)\right] s\left[z_{l}(\sigma)-L_{l}(\xi, \sigma)\right] \\
= & K_{1}+K_{2} .
\end{aligned}
$$

As in the estimate of $\zeta K_{2}$ in step (iii)

$$
\left|E\left\{\zeta K_{2}\right\}\right| \leqq C_{25} E^{1 / 4}\left\{\zeta^{4}\right\} \frac{1}{\varepsilon} \int_{t}^{u} d \sigma \frac{(\sigma-\xi)^{4}}{\varepsilon^{6}} \rightarrow 0
$$


as $\varepsilon \downarrow 0$ (use (3.89)). $K_{1}$ is rewritten by means of (3.43) as

$$
\begin{aligned}
& \frac{1}{\varepsilon^{4}} \int_{t}^{u} d \sigma \int_{\xi}^{\sigma} d \lambda \int_{\xi}^{\lambda} d \rho \frac{\partial f}{\partial w_{i}}(w(\xi)) D_{2, j} G_{i}(\sigma, L(\xi, \sigma), w(\xi)) \\
& \cdot G_{j}(\rho, z(\rho), w(\rho)) \\
& =\frac{1}{\varepsilon^{4}} \int_{t}^{u} d \sigma \int_{\xi}^{\sigma} d \lambda \int_{\xi}^{\lambda} d \rho \frac{\partial f}{\partial w_{i}}(w(\xi)) D_{2, j} G_{i}(\sigma, L(\xi, \sigma), w(\xi)) \\
& \cdot G_{j}(\rho, z(\rho), w(\xi)) \\
& \quad+\frac{1}{\varepsilon^{5}} \int_{t}^{u} d \sigma \int_{\xi}^{\sigma} d \lambda \int_{\xi}^{\lambda} d \rho \frac{\partial f}{\partial w_{i}}(w(\xi)) D_{2, j} G_{i}(\sigma, L(\xi, \sigma), w(\xi)) \\
& \cdot \int_{\xi}^{\rho} d \tau D_{3, l} G_{j}(\rho, z(\rho), w(\tau)) G_{l}(\tau, z(\tau), w(\tau)) \\
& =L_{1}+L_{2} .
\end{aligned}
$$

By Holder's inequality and Lemma 3,

$$
\begin{aligned}
\left|E\left\{\zeta L_{2}\right\}\right| & =O\left(\frac{1}{\varepsilon^{5}} \int_{t}^{u} d \sigma \int_{\xi}^{\sigma} d \lambda \int_{\xi}^{\lambda} d \rho \int_{\xi}^{\rho} d \tau\right)=O\left(\int_{t}^{u} d \sigma \frac{(\sigma-\xi)^{3}}{\varepsilon^{5}}\right) . \\
& =o(1) \quad \text { as } \varepsilon \downarrow 0 .
\end{aligned}
$$

Similarly we may in $L_{1}$ replace $G_{j}(\rho, z(\rho), w(\xi))$ by $G_{j}(\rho, L(\xi, \rho), w(\xi))$. So far we have shown

$$
\begin{aligned}
E\left\{\zeta_{1}\right\}= & o(1)+\frac{1}{\varepsilon^{4}} E\left\{\int_{t}^{u} d \sigma \int_{\xi}^{\sigma} d \lambda \int_{\xi}^{\lambda} d \rho \frac{\partial f}{\partial w_{i}}(w(\xi))\right. \\
& \left.\cdot D_{2, j} G_{i}(\sigma, L(\xi, \sigma), w(\xi)) G_{j}(\rho, L(\xi, \rho), w(\xi)) \zeta\right\} .
\end{aligned}
$$

We now apply (for the first time) Lemma 5. We obtain with (we still use the summation conventions)

$$
\begin{aligned}
V_{i}\left(y, w_{1}, z, w_{2}\right) & =E\left\{F_{j}\left(y, w_{1}\right) \frac{\partial}{\partial z_{j}} F_{i}\left(z, w_{2}\right)\right\}, \\
W_{i j}\left(y, w_{1}, z, w_{2}\right) & =E\left\{F_{j}\left(y, w_{1}\right) F_{i}\left(z, w_{2}\right)\right\},
\end{aligned}
$$

that

$$
\begin{aligned}
E\left\{\zeta I_{1}\right\}= & o(1)+\frac{1}{\varepsilon^{4}} E\left\{\int_{t}^{u} d \sigma \int_{\xi}^{\sigma} d \lambda \int_{\xi}^{\lambda} d \rho \frac{\partial f}{\partial w_{i}}(w(\xi)) \zeta \Psi(\rho, w(\xi))\right. \\
& . \Psi(\sigma, w(\xi)) \Phi(\rho, L(\xi, \rho))\left[\Phi(\sigma, L(\xi, \sigma)) V_{i}(L(\xi, \rho), w(\xi), L(\xi, \sigma), w(\xi))\right. \\
& +\frac{\partial \Phi}{\partial z_{j}}\left(\sigma, L(\xi, \sigma) W_{i j}(L(\xi, \rho), w(\xi), L(\xi, \sigma), w(\xi))\right] \\
& +O\left(\frac{1}{\varepsilon^{4}} \int_{t}^{u} d \sigma \int_{\xi}^{\sigma} d \lambda \int_{\xi}^{\lambda} d \rho\left\{\varepsilon^{2}+\gamma\left(\frac{\rho-\xi}{\varepsilon^{2}}\right)\right\}^{1 / 2}\left\{\varepsilon^{2}+\gamma\left(\frac{\sigma-\rho}{\varepsilon^{2}}\right)\right\}^{1 / 2}\right) .
\end{aligned}
$$


The last error term is again $o(1)$ by (3.71) and (3.89). Also, by (3.10), (3.79) and (3.77)

$$
\left|\frac{\partial \phi}{\partial z_{j}}(\sigma, L(\xi, \sigma)) W_{i j}(L(\xi, \rho), w(\xi), L(\xi, \sigma), w(\xi))\right| \leqq C_{26^{2}} \varepsilon^{2}\left\{\beta\left(\frac{\sigma-\rho}{2 N \varepsilon^{2}}\right)\right\}^{1 / 2} .
$$

and this term too can be dropped. This almost gives us the required form. We still observe that for $k \eta \leqq t \leqq \xi \leqq \rho \leqq \sigma \leqq u<(k+1) \eta$

$$
\Psi(\rho, w(\xi))=\Psi(\sigma, w(\xi))=\Psi(\xi, w(\xi)) .
$$

Similarly, by (3.10) and (3.11),

$$
\begin{aligned}
& |\Phi(\rho, L(\xi, \rho))-\Phi(\xi, z(\xi))|=\mid \Phi(\xi, L(\xi, \rho)-\Phi(\xi, z(\xi)) \mid \\
& \quad \leqq C_{1} \varepsilon^{2}|L(\xi, \rho)-z(\xi)|=C_{1}(\rho-\xi)|w(\xi)| \\
& \quad \leqq 2 C_{1} M(\rho-\xi),
\end{aligned}
$$

and the same inequality holds when $\rho$ is replaced by $\sigma$ throughout. From these observations, (3.79) and (3.10) we see that

$$
\begin{aligned}
E\left\{\zeta I_{1}\right\}= & o(1)+\int_{t}^{u} d \sigma E\left\{\frac{\partial f}{\partial w_{i}}(w(\xi)) \zeta \Psi^{2}(\xi, w(\xi)) \phi^{2}(\xi, z(\xi))\right. \\
& \left.\cdot \frac{1}{\varepsilon^{4}} \int_{\xi}^{\sigma} d \rho(\sigma-\rho) V_{i}(L(\xi, \rho), w(\xi), L(\xi, \sigma), w(\xi))\right\} .
\end{aligned}
$$

Last, if we take into account the stationarity of $F$ (see (II)),

$$
\begin{aligned}
& \Psi(\xi, w(\xi)) \frac{1}{\varepsilon^{4}} \int_{\xi}^{\sigma} d \rho(\sigma-\rho) V_{i}(L(\xi, \rho), w(\xi), L(\xi, \sigma), w(\xi)) \\
& \quad=\Psi(\xi, w(\xi)) \int_{0}^{\varepsilon^{-2}(\sigma-\xi)} t E\left\{F_{j}(0, w) \frac{\partial}{\partial z_{j}} F_{i}(t w, w)\right\}_{w=w(\xi)} d t \\
& \quad=\sum_{j} c_{i j}(\xi, w(\cdot), w(\xi))+o\left(\int_{\varepsilon^{-2}(\sigma-\xi)}^{\infty} t \beta^{1 / 2}(t|w(\xi)|) d t\right) \quad(\text { by }(3.79)) \\
& =\sum_{j} c_{i j}(\xi, w(\cdot), w(\xi))+o(1) \quad(\text { by }(3.89),(3.57) \text { and (3.11)). }
\end{aligned}
$$

Thus

$$
\begin{aligned}
E\left\{\zeta I_{1}\right\}= & o(1)+\int_{t}^{u} d \sigma E\left\{\frac{\partial f}{\partial w_{i}}(w(\xi)) \zeta \Psi(\xi, w(\xi)) \Phi^{2}(\xi, z(\xi))\right. \\
& \cdot \sum_{j} c_{i, j}(\xi, w(\cdot), w(\xi)) . \\
= & o(1)+\int_{t}^{u} d \xi E\left\{\frac{\partial f}{\partial w_{i}}(w(\xi)) \zeta \Psi(\xi, w(\xi)) \Phi^{2}(\xi, z(\xi)) \sum_{j} c_{i, j}(\xi, w(\cdot), w(\xi)) .\right.
\end{aligned}
$$

The last equality results merely from the fact that $\sigma=\xi+\varepsilon^{15 / 8}$ except on an interval of length $\varepsilon^{15 / 8}$. Finally, we view $w(\cdot)$ as an element of $D$. Then the expectation in the last integral can be written as an integral over $D$ with respect to the 
measure $R^{\varepsilon}(\cdot ; M, N, 1 / p, 1 / q)$ and integrand

$$
\frac{\partial f}{\partial w_{i}}(X(\xi)) \zeta(X(\cdot)) \Psi_{*}(\xi, X(\cdot)) \Phi_{*}^{2}(\xi, X(\cdot)) \sum_{j} c_{i j}(\xi, X(\cdot), X(\xi))
$$

(This is where we use (3.86)). As observed already in [6] it follows from (3.81) and (3.84) that for each fixed $\sigma, X(\cdot)$ is continuous at $\sigma$ with $R(\cdot ; M, N, 1 / p, 1 / q)$ probability one. From this one easily derives that (3.90) is a continuous function of $X(\cdot)$ a.e. on $D$ with respect to $R(\cdot ; M, N, 1 / p, 1 / q)$ (in the usual $J_{1}$ topology). Thus, if we write $E^{M, N, p, q}$ for the expectation operator with respect to $R(\cdot ; M, N, 1 / p, 1 / q)$ and let $\varepsilon \rightarrow 0$ through the sequence $\varepsilon_{n}$, then (3.84) yields

$$
\begin{aligned}
\lim _{n \rightarrow \infty} E\left\{\zeta I_{1}\right\}= & \int_{t}^{u} d \xi E^{M, N, p, q}\left\{\zeta(X(\cdot)) \Psi_{*}(\xi, X(\cdot)\}\right. \\
& \cdot \Phi_{*}^{2}(\xi, X(\cdot)) \sum_{j} c_{i j}(\xi, X(\cdot), X(\xi)) \frac{\partial f}{\partial w_{i}}(X(\xi)) .
\end{aligned}
$$

which is one of the terms of the right-hand side of (3.88). The other terms come from $E\left\{\zeta_{I_{2}}\right\}$. This proves the claimed martingale property for (3.85).

\section{Step $(v)$}

We complete the proof of our theorem in this step by removing the truncations in $N, \eta, \delta$ and $M$ (in this order). It is convenient to return first to the space $C=$ $C\left([0, \infty) ; \mathbb{R}^{d}\right)$. We assumed that the convergence in (3.84) takes place on $D$. However, by definition $R^{\varepsilon}(\cdot ; M, N, 1 / p, 1 / q)$ is concentrated on $C$, and if we can show that $R(\cdot ; M, N, 1 / p, 1 / q)$ is also concentrated on $C$ then (3.84) implies

$$
R^{\varepsilon_{n}}\left(\cdot ; M, N, \frac{1}{p}, \frac{1}{q}\right) \Rightarrow R\left(\cdot ; M, N, \frac{1}{p}, \frac{1}{q}\right) \text { on } C
$$

(see [8], p. 151). But the fact that $R(\cdot ; M, N, 1 / p, 1 / q)$ is concentrated on $C$ follows immediately from the martingale property of (3.85), Theorem 2.1 (especially inequality (2.1)) of [15] and the fact that

$$
\begin{aligned}
& \psi_{*}(\sigma, X) \Phi_{*}^{2}(\sigma, X)\left\{\left|\psi_{*}(\sigma, X) a_{i j}(X(\sigma))\right|+\left|b_{i}^{M, N, p}(X(\sigma))\right|\right\} \\
& \quad \leqq \sup _{1 / 2 M \leqq|v| \leqq 2 M}\left\{\left|a_{i j}(v)\right|+\left|b_{i}^{M, N, p}(v)\right|\right\}<\infty
\end{aligned}
$$

(because $\Psi_{*}(\sigma, X)=0$ for $|X(\sigma)| \notin[1 / 2 M, 2 M]$; see (3.1)).

From now on we can assume (3.91) and all further manipulations take place on $C$. With a slight abuse of notation we also use $X_{t}$ for the $t$-coordinate function on $C$ and $\mathscr{M}_{v}^{u}$ for $\sigma\left\{X_{t}: u \leqq t \leqq v\right\}$. Formula (3.85) still is an $\left(R(\cdot ; M, N, 1 / p, 1 / q), M_{t}^{0}\right)$ martingale, even with the convention that $R$ is a measure on $\mathscr{M}_{\infty}^{0}$ and $\mathscr{M}_{t}^{0}, \mathscr{M}_{\infty}^{0}$ $\sigma$-fields in $C$. We now define $\left\{\mathscr{M}_{t}^{0}\right\}$ stopping times, $S, T, U$ and $V$ as follows:

$$
S=S(X(\cdot) ; N, p)=\lim _{n \rightarrow \infty} S_{n},
$$


where $X_{k}$ is as in step (i) with $\eta=1 / p$ and

$$
S_{n}=\inf \left\{t \geqq 0: \text { for some } k \geqq 0, \frac{k}{p} \leqq t<\frac{k+1}{p}\right.
$$

and

$$
\left.\left(X_{k-1}, X(t)\right)<\frac{1}{N}+\frac{1}{n} \text { or }\left(X_{k}, X_{t}\right)<\frac{1}{N}+\frac{1}{n}\right\} .
$$

$S_{n}$ is an $\left\{\mathscr{M}_{t^{+}}^{0}\right\}$ stopping time and for $t$ not of the form $k / p$

$$
\{S \leqq t\}=\bigcap_{n}\left\{S_{n}<t\right\} \in \mathscr{M}_{t}^{0} .
$$

For $t=k / p$.

$$
\{S \leqq t\}=\left(\bigcap_{n}\left\{S_{n}<t\right\}\right) \cup\left(\left(X(t), X_{k}\right) \leqq \frac{1}{N}\right) \in \mathscr{M}_{t}^{0}
$$

so that indeed $S$ is an $\mathscr{M}_{t}^{0}$ stopping time. Similarly,

$$
\begin{aligned}
T & =T(X(\cdot) ; M))=\lim _{n \rightarrow \infty} T_{n}, \\
T_{n} & =\inf \left\{t \geqq 0:|X(t)|<\frac{1}{M}+\frac{1}{n} \text { or }|X(t)|>M-\frac{1}{n}\right\}, \\
U & =U(X(\cdot) ; p, q)=\lim _{n \rightarrow \infty} U_{n}, \\
U_{n} & =\inf \{t \geqq 0: \text { for some } k \geqq 1 \text { and } u \leqq(k-1) p, k / p \leqq t<(k+1) p \text { and } \\
\left.\left|\int_{u}^{t} X(v) d v\right|<\frac{1}{q}+\frac{1}{n}\right\} &
\end{aligned}
$$

Lastly,

$$
V=S \wedge T \wedge U
$$

From these definitions it follows that

$$
\Psi_{*}(\sigma, X)=1 \text { and } \frac{\partial}{\partial w_{j}} \Psi(\sigma, X, X(\sigma))=0 \quad \text { for } \sigma<S \vee T
$$

and

$$
\Phi_{*}(\sigma, X)=1 \quad \text { for } \sigma<U .
$$

Thus

$$
\begin{gathered}
f\left(X_{t \wedge V}\right)-\int_{0}^{t \wedge V}\left(L^{M, N, p, q} f\right)(\sigma, X) d \sigma \\
=f\left(X_{t \wedge V}\right)-\int_{0}^{t \wedge V} \mathscr{L} f(X(\sigma)) d \sigma \\
=f\left(X_{t \wedge V}\right)-\int_{0}^{t \wedge V} \tilde{\mathscr{L}}^{M} f(X(\sigma)) d \sigma,
\end{gathered}
$$


where $\mathscr{L}$ is given by $(2.5)$ and

$$
\tilde{\mathscr{L}}^{M} f(v)=\frac{1}{2} \sum_{i, j} \tilde{a}_{i j}^{M}(v) \frac{\partial^{2}}{\partial v_{i} \partial v_{j}} f(v)+\sum_{i} \tilde{b}_{i}^{M}(v) \frac{\partial f}{\partial v_{i}}(v)
$$

for any choice of the coefficients $\tilde{a}_{i j}^{M}(v), \tilde{b}_{i}^{M}(v)$ which agrees with the $a_{i j}(v)$ and $b_{i}(v)$ of $(2.3),(2.4)$ on the set $\left\{\frac{1}{M} \leqq|v| \leqq M\right\}$ and which makes the $\tilde{a}_{i j}^{M}$ and $\tilde{b}_{i}^{M}$ twice continuously differentiable on $\mathbb{R}^{d}$ and $\tilde{a}_{i j}^{M}$ strictly positive definite.

Now let $\widetilde{Q}^{M}$ be the measure corresponding to the diffusion process $\widetilde{\mathscr{L}}^{M}$ and initial point $v_{0}$, and let $Q$ be as in the theorem. Then (3.92) is an $\left(R(\cdot ; M, N, p, q), \mathscr{M}_{t}^{0}\right)$ martingale, by virtue of the optional sampling theorem (cf. [14], Theorem VI.7 and 13) and the martingale property of (3.85). (3.92) is also a $\left(Q, \mathscr{M}_{t}^{0}\right)$ and a $\left(\widetilde{Q}^{M}, \mathscr{M}_{t}^{0}\right)$ martingale by Dynkin's formula ([11] Corollary 5.1). Moreover, by (3.91)

$$
Q(X(0)=0)=\widetilde{Q}^{M}\left(X(0)=v_{0}\right)=R\left(X(0)=v_{0} ; M, N, p, q\right)=1
$$

We claim that this implies

$$
\begin{gathered}
\widetilde{Q}^{M}(B)=Q(B)=R(B ; M, N, p, q) \text { whenever } \\
B \in \mathscr{M}_{V(M, N, p, q)}^{0} ;
\end{gathered}
$$

(see [16] for the definition of the last $\sigma$-field). Indeed if $v$ is any of the measures $\widetilde{Q}^{M}, Q$ or $R(\cdot ; M, N, p, q)$ then one can use Lemma 3.6 and Theorem 3.4 of [16] and the martingale property of (3.92) to construct a measure $\mu$ on $\mathscr{M}_{\infty}^{0}$ which agrees with $v$ on $\mathscr{M}_{V}^{0}$ and such that

$$
f\left(X_{t}\right)-\int_{0}^{t} \tilde{\mathscr{L}}^{M} f(X(\sigma)) d \sigma
$$

is a $\left(\mu, \mathscr{M}_{t}^{0}\right)$ martingale and (by (3.93)).

$$
\mu(X(0)=0)=1 .
$$

By the uniqueness theorem 6.2 of [16] such a $\mu$ is unique. Thus all three choices for $v$ give rise to the same $\mu$ which implies (3.94).

We apply (3.94) to

$$
B=B(M, N, p, q)=\{S(N, p) \wedge U(p, q) \leqq T(M) \wedge t\}
$$

for fixed $t$. This gives

$$
R(B(M, N, p, q) ; M, N, p, q)=Q(B(M, N, p, q)) .
$$

We shall show in Lemma 6, Section 4 that as a consequence of assumption $V$, for each fixed $p$,

$$
\lim _{q \rightarrow \infty} U(p, q)=\infty \text { a.e. }[Q]
$$

This of course implies

$$
\lim _{q \rightarrow \infty} Q\{U(p, q) \leqq T \wedge t\}=0
$$


In addition, for $N \geqq 2 M$

$$
\lim _{p \rightarrow \infty} Q\{S(N, p) \leqq T(M) \wedge t\}=0
$$

because $|X(\sigma)| \geqq 1 / M$ for all $\sigma \leqq T(M)$ and $X(\cdot)$ is continuous. It follows that the right-hand side of (3.95) can be made small for any $N \geqq 2 M$ by choosing first $p$, then $q$ large.

It is now easy to remove the cutoffs. Fix $t$ and any bounded positive continuous functional $f$ on $C$ which is measurable with respect to $\mathscr{M}_{t}^{0}$. Let $\varepsilon_{n} \rightarrow 0$, and if necessary refine the sequence such that

$$
\lim _{n \rightarrow \infty} E\left\{f\left(v^{\varepsilon_{n}}(\cdot)\right) ; T\left(v^{\varepsilon_{n}}, M\right)>t\right\}
$$

exists for all integers $M$, and such that (3.91) holds for all integers $M, N, p$ and $q$. Here we view $v^{\varepsilon}$ as an element of $C$ and $T\left(v^{\varepsilon}, M\right)$ is the value of $T(M)$ evaluated at $v^{\varepsilon}$. Fix $M$ and $\alpha>0$ and choose $N, p, q$ such that

$$
Q(\bar{B}(M-1, N-1, p, q-1)) \leqq Q(B(M, N-2, p, q-2)) \leqq \alpha
$$

and

$$
Q(B(M, N, p, q)) \leqq \alpha
$$

$\left(\bar{B}\right.$ denotes the closure of $B$ in $C$.) Finally note that $v^{\varepsilon}(t)=w^{\varepsilon}(t ; M, N, p, q)$ for all

$$
t \leqq V\left(v^{\varepsilon} ; M, N, p, q\right)=V\left(w^{\varepsilon} ; M, N, p, q\right)
$$

because the Eqs. (2.7) for $v^{\varepsilon}$ and (3.8) for $w^{\varepsilon}$ coincide up until this time $V$. This, together with (3.91) and (3.94), implies that

$$
\begin{aligned}
& \limsup _{n \rightarrow \infty} E\left\{f\left(v^{\varepsilon_{n}}(\cdot)\right) ; V\left(v^{\varepsilon_{n}} ; M-1, N-1, p, q-1\right)>t\right\} \\
& \quad=\limsup _{n \rightarrow \infty} E\left\{f\left(w^{\varepsilon_{n}}(\cdot)\right) ; V\left(w^{\varepsilon_{n}} ; M-1, N-1, p, q-1\right)>t\right\} \\
& \quad \leqq E^{M, N, p, q}\{f(X(\cdot)) ; V(X ; M, N, p, q)>t\} \\
& \quad=\int f(X(\cdot)) I[V(X ; M, N, p, q)>t] d Q .
\end{aligned}
$$

Moreover, by our choice of $N, p, q$, the last member here differs from

$$
\int f(X(\cdot)) I[T(X, M)>t] d Q
$$

by at most

$$
\sup |f| Q(B(M, N, p, q)) \leqq \alpha \sup |f| .
$$

Finally,

$$
\begin{aligned}
& \limsup _{n \rightarrow \infty} \mid E\left\{f\left(v^{\varepsilon_{n}}(\cdot)\right) ; T\left(v^{\varepsilon_{n}}, M-1\right)>t\right\} \\
& -E\left\{f\left(v^{\varepsilon_{n}}(\cdot)\right) ; V\left(v^{\varepsilon_{n}} ; M-1, N-1, p, q-1\right)>t\right\} \mid \\
& \leqq \sup |f| \limsup _{n \rightarrow \infty} P\left\{S\left(v^{\varepsilon_{n}} ; N-1, p\right) \wedge U\left(v^{n} ; p, q-1\right)\right. \\
& \left.\leqq T\left(v^{\varepsilon_{n}} ;(M-1) \wedge t\right)\right\},
\end{aligned}
$$


and (again by (3.97), (3.91) and (3.94)), the lim sup in the right-hand side of (3.98) equals

$$
\begin{aligned}
& \limsup _{n \rightarrow \infty} P\left\{S\left(w^{\varepsilon_{n}} ; N-1, p\right) \wedge U\left(w^{\varepsilon_{n}} ; p, q-1\right) \leqq T\left(w^{\varepsilon_{n}} ;(M-1) \wedge t\right)\right\} \\
& \quad=\limsup _{n \rightarrow \infty} \mathrm{R}^{\varepsilon_{n}}(B(M-1, N-1, p, q-1) ; M, N, p, q) \\
& \quad \leqq R(\bar{B}(M-1, N-1, p, q-1) ; M, N, p, q) \\
& \quad=Q(\bar{B}(M-1, N-1, p, q-1)) \leqq \alpha .
\end{aligned}
$$

Since $\alpha$ is arbitrary these estimates show that for each $M, t$,

$$
\begin{aligned}
& \limsup _{n \rightarrow \infty} E\left\{f\left(v^{\varepsilon_{n}}(\cdot)\right) ; T\left(v^{\varepsilon_{n}}, M-1\right)>t\right\} \\
& \quad \leqq \int f(X(\cdot)) I[T(X, M)>t] d Q .
\end{aligned}
$$

Finally, we take the limit as $M \rightarrow \infty$. From (3.99) we see that

$$
\lim \sup P\left\{T\left(v^{\varepsilon_{n}}, M-1\right)>t\right\} \leqq Q\{T(X, M)>t\}
$$

and this can be made as small as desired by taking $M$ large, on account of assumption (2.6) and the boundedness of $a_{i j}(\cdot)$ and $b_{i}(\cdot)$ away from the origin (see [15], formula (2.1)). Thus

$$
\limsup _{n \rightarrow \infty} E\left\{f\left(v^{\varepsilon_{n}}(\cdot)\right)\right\} \leqq \int f(X(\cdot)) d Q .
$$

Since any sequence $\varepsilon_{n} \rightarrow 0$ contains a subsequence to which this applies we have proved

$$
\lim \sup E\left\{f\left(v^{\varepsilon}(\cdot)\right)\right\} \leqq \int f(X(\cdot)) d Q
$$

As shown in [8], p. 13, and [6], Sect. 3, Step (vi), this implies that the measures induced by $v^{\varepsilon}(\cdot)$ on $C$ converge weakly to $Q$ on $C$. The proof is complete.

\section{Properties of Diffusions and Examples}

We begin this section with Lemma 6 which immediately implies the relation (3.96) which we needed in Step (iv) of Sect. 3. The proof of this lemma is entirely independent of Sect. 3.

Lemma 6. Let $V_{t}$ be a diffusion with generator $\mathscr{L}$ as in(2.3)-(2.5) and initial point $v_{0} \neq 0$. Assume that $\left(a_{i j}(\cdot)\right)$ is symmetric and strictly positive definite on $\mathbb{R}^{d} \backslash\{0\}$ and

$$
a_{i j}(\cdot), b_{i}(\cdot) \in C^{\infty}\left(\mathbb{R}^{d} \backslash\{0\}\right) .
$$

If $d \geqq 3, v_{0} \neq 0$ and (2.6) holds, then for all $\eta>0, T<\infty$,

$$
\lim _{\delta \downarrow 0} P^{v_{0}}\left\{\left|\int_{s}^{t} V_{\sigma} d \sigma\right|<\delta \text { for some } 0 \leqq s, t \leqq T \text { with }|t-s| \geqq \eta\right\}=0 .
$$

Remark 5. A check of the proof in the previous section shows that the only place 
where the smoothness of the $a_{i j}$ and $b_{i}$ and the strict positive definiteness of $a_{i j}$ played a role was in the proof of (3.94), and more precisely for the uniqueness of the measure $\mu$ in that proof. For this uniqueness we only need

$\left(a_{i j}(\cdot)\right)$ is nonnegative definite and twice continuously differentiable on $\mathbb{R} \backslash\{0\} ;$

(see [17], Theorem 2.3 and Remark 2.1; note that the $b_{i}(v)$ of (2.4) is uniformly Lipschitz continuous and bounded on any set $\left\{v \in \mathbb{R}^{d}:|v| \geqq \delta\right\}, \delta>0$, by (II)-(IV)). The stronger requirement (4.1) and the requirement $d \geqq 3$ are used only to prove Lemma 6. Therefore, our theorem and corollary will remain valid in any dimension if in (V) we only require (4.3) of the coefficients and (2.6), provided we add (4.2) as a separate hypothesis. Actually, it is likely that even for Lemma 6 only a finite number of derivatives of $a_{i j}(\cdot)$ and $b_{i}(\cdot)$ are needed, but we have not pursued this.

The proof of Lemma 6 will come after we discuss a sufficient condition for (2.6), and (III) and explicit examples. Formula (2.6) can often be verified by means of the following criterion of Khasminskii's [18] (see also [9], Chap. 4.5).

Lemma 7. Let $\mathscr{L}$ and $V_{t}$ be as in $(V)$. For $v \neq 0$ and $\Gamma=\left(\Gamma_{i j}\right)$ a nonsingular (constant) $d \times d$ matrix define $\left(\Gamma^{t}=\right.$ transpose of $\left.\Gamma\right) A(v)=A(v, \Gamma)=\sum_{i, j}\left(\Gamma a(v) \Gamma^{t}\right)_{i, j}(\Gamma v)_{i}(\Gamma v)_{j}$,

$$
\begin{aligned}
A^{+}(r, \Gamma) & =\max _{|\Gamma v|} A(v, \Gamma), \\
B_{-}(r) & =B_{-}(r, \Gamma) \\
& =\min _{|\Gamma v|=r} \frac{1}{A(v, \Gamma)}\left[2 \sum_{i}(\Gamma b(v))_{i}(\Gamma v)_{i}+\sum_{i}\left(\Gamma a(v) \Gamma^{t}\right)_{i, i}\right],
\end{aligned}
$$

and

$$
C_{-}(r, \Gamma)=\exp \left\{-\int_{r}^{1} s B_{-}(s, \Gamma) d s\right\} .
$$

If there exists a nonsingular $\Gamma$ such that

$$
\int_{0}^{1} \frac{t d t}{C_{-}(t, \Gamma)} \int_{t}^{1} \frac{C_{-}(s, \Gamma)}{A_{+}(s, \Gamma)} d s=\infty,
$$

then (2.6) holds.

Remark 6. Take $d \geqq 3$ and

$$
\mathscr{L}=\sum_{i, j} \frac{\partial}{\partial v_{i}} \frac{a_{i j}}{|v|} \frac{\partial}{\partial v_{j}}
$$

for some constant symmetric positive definite matrix $\alpha$, as discussed in Remark 4. Then (4.4) holds for $\Gamma=\alpha^{-1 / 2}$ (the positive symmetric square root), so that also (2.6) holds in this case. In general the criterion of Lemma 7 is only helpful if for some $\Gamma$ the diffusion $\left\{\Gamma V_{t}\right\}_{t \geqq 0}$ is close to being radially symmetric (see the examples in [9], Chap. 4.5).

Lemma 7 too will be proved later. First we discuss two situations which 
guarantee the mixing conditions (III). (a) (III) holds for what we called the trivial Gaussian examples in [6]. These are Gaussian fields $\{F(x, v)\}_{x, v \in \mathbb{R}^{d} \times \mathbb{R}^{d}}$ with mean zero, stationary in $x$, and such that the correlation function

$$
r_{i j}\left(y, v_{1}, v_{2}\right)=E\left\{F_{i}\left(x, v_{1}\right) F_{j}\left(x+y, v_{2}\right\}\right.
$$

vanishes for all $i, j, v_{1}, v_{2}$ as soon as $|y| \geqq L$ where $L<\infty$ is some constant. In this case $\mathscr{G}_{\Lambda_{1}}$ and $\mathscr{G}_{\Lambda_{2}}$ are actually independent as soon as $d\left(\Lambda_{1}, \Lambda_{2}\right)>L$. Thus $\beta(\rho)=0$ for $\rho>L$ and (2.2) certainly holds. (It is not hard to show that for these stationary Gaussian fields it is actually necessary for $(2.2)$ that $\beta(\rho)$ vanishes for large $\rho$ ). One can obtain such examples by taking ( $\bar{\rho}$ is the complex conjugate of $\rho$ )

$$
r_{i j}\left(y, v_{1}, v_{2}\right)=\sum_{k} \int_{\mathbb{R}^{d}} \rho_{i, k}\left(y+z, v_{1}\right) \bar{\rho}_{j, k}\left(z, v_{2}\right) d z
$$

for any measurable complex matrix valued function $\rho$ on $\mathbb{R}^{d} \times \mathbb{R}^{d}$ with $\rho(z, v)=0$ for $|z| \geqq L / 2$, as long as

$$
\int_{\mathbb{R}^{d}}\left|\rho_{i i}(z)\right|^{2} d z<\infty \text { for all } i .
$$

It is easy to see that (4.6) is indeed positive definite, hence a correlation function, and has support in $|y| \leqq L$. This leads to the following explicit example: The Theorem and Corollary apply if $d \geqq 3$ and $\{F(x, v)\}$ is a mean zero Gaussian field independent of $v$ and stationary in $x$, with correlation function given by (4.5) and (4.6) with

$$
\rho(y, v)=\rho^{*}(y)=\rho^{*}(|y|)
$$

(depending on $|y|$ only), whenever $\rho^{*} \in C^{2}\left(\mathbb{R}^{d}\right)$ and the following properties hold:

$$
\begin{aligned}
& \rho^{*}(z)=0 \text { for }|z| \geqq \frac{1}{2} L . \\
& \int_{\mathbb{R}^{d}}\left|\left(\frac{\partial}{\partial z_{l}}\right)^{2} \rho_{i i}^{*}(z+u)-\left(\frac{\partial}{\partial z_{l}}\right)^{2} \rho_{i i}^{*}(z)\right| d z \leqq K\left(\log \frac{1}{|\eta|}\right)^{\alpha}
\end{aligned}
$$

for some $K<\infty, \alpha>1$ and all $|u| \leqq 1,1 \leqq i, l \leqq d$, and finally,

$$
\begin{aligned}
\alpha_{i j}= & \sum_{k} \int_{0}^{\infty} d t \int_{\mathbb{R}^{d}} \rho_{i k}^{*}(t e+z) \overline{\rho_{j, k}^{*}}(z) d z \\
& \text { is nonsingular for } e=(1,0, \ldots, 0) .
\end{aligned}
$$

In this case

$$
\mathscr{L}=\sum_{i, j} \alpha_{i j} \frac{\partial}{\partial v_{i}} \frac{1}{|v|} \frac{\partial}{\partial v_{j}} .
$$

See below for a proof.

(b) (III) also holds for the following special case of the "Poisson blobs" of [6]: Let $P_{\rho}$ be a Poisson point process on $\mathbb{R}^{d}$ with intensity $\rho$, i.e. $N(B) \equiv$ number of points of $P_{\rho}$ in the Borel set $B \subset \mathbb{R}^{d}$ has a Poisson distribution with mean $\rho|B|$ 
$(|B|$ denotes the Lebesgue measure of $B)$. Moreover, if $B_{1}, \ldots, B_{k}$ are disjoint, then $N\left(B_{1}\right), \ldots, N\left(B_{k}\right)$ are independent. In addition, let $H^{0}, H^{1}, H^{2}, \ldots$ be independent identically distributed random fields, independent of $P_{\rho}$ and with the following properties:

Each $H^{(n)}$ is indexed by $\mathbb{R}^{d}$, i.e. $H^{(n)}=H^{(n)}(x, \omega), x \in \mathbb{R}^{d}, \omega$ in our basic probability space. $H^{(0)}(x)=0$ for $|x| \geqq L$ for some constant $L$.

Let

$$
K(x)=\sum_{n=1}^{\infty} H^{(n)}\left(x+p_{n}\right)
$$

where $p_{1}, p_{2}, \ldots$ are the (random) points of $P_{\rho}$, and let $f$ be some deterministic function from $\mathbb{R}^{d} \times \mathbb{R}^{d}$ to $\mathbb{R}^{d}$. Then the field

$$
F(x, v)=f(K(x), v)
$$

satisfies the mixing condition (III). Again we can make this into an explicit example. Assume that $H^{(0)}$ also has the following properties:

The joint distribution of $H^{(0)}\left(x_{1}\right)$ and $H^{(0)}\left(x_{2}\right)$ is the same as that of $H^{(0)}\left(O x_{1}\right)$ and $H^{(0)}\left(O x_{2}\right)$ for any $x_{1}, x_{2} \in \mathbb{R}^{d}$ and orthogonal matrix $O$.

$$
E\left\{H^{(0)}(x)\right\}=0, \quad x \in \mathbb{R}^{d}
$$

$H^{(0)}$ is twice continuously differentiable and

$$
E\left\{\max _{|x| \leqq L}\left|D^{\beta} H^{(0)}(x)\right|^{p}\right\} \leqq c_{0},
$$

for $|\beta| \leqq 2, p \leqq 16 d+64$.

The $d \times d$ matrix with entries

$$
\alpha_{i j}=\rho \int_{0}^{\infty} d t \int_{\mathbb{R}^{d}} E\left\{H_{i}^{(0)}(z) H_{j}^{(0)}(t e+z)\right\} d z,
$$

is nonsingular $(e=(1,0,0, \ldots, 0))$.

Then our Theorem and Corollary apply if we take $d \geqq 3$ and

$$
F(x, v)=\sum_{n} H^{(n)}\left(x+p_{n}\right)
$$

(independent of $v$; corresponding to $f(K, v)=K$ in (4.12)).

In this case

$$
\mathscr{L}=\sum_{i, j} \alpha_{i, j} \frac{\partial}{\partial v_{i}} \frac{1}{|v|} \frac{\partial}{\partial v_{j}} \text {. }
$$

We turn to the proofs.

Proof of Lemma 6. We are indebted to Daniel W. Stroock for pointing out how to use hypoellipticity in the first part of this proof. Let

$$
Z_{t}=\int_{0}^{t} V_{\sigma} d \sigma
$$


Then $\left(V_{t}, Z_{t}\right)_{t \geqq 0}$ is a singular diffusion with generator

$$
\mathscr{L}+\sum_{i=1}^{d} v_{i} \frac{\partial}{\partial z_{i}}
$$

Since the coefficients of $\mathscr{L}$ may be badly behaved at the origin we first replace $\mathscr{L}$ by

$$
\tilde{\mathscr{L}}^{M}=\frac{1}{2} \sum_{i, j} \tilde{a}_{i j}^{M}(v) \frac{\partial^{2}}{\partial v_{i} \partial v_{j}}+\sum_{i} \tilde{b}_{i}^{M}(v) \frac{\partial}{\partial v_{i}}
$$

for any choice of the coefficients $\tilde{a}_{i j}^{M}(x), \tilde{b}_{i}^{M}(x)$ which agrees with the $a_{i j}(v)$ and $b_{i}(v)$ of $\mathscr{L}$ on the $\operatorname{set}\left\{\frac{1}{M} \leqq|v| \leqq M\right\}$ and which makes $\tilde{a}_{i j}^{M}$ and $\tilde{b}_{i}^{M}$ infinitely differentiable on $\mathbb{R}^{d}$ and $\tilde{a}_{i j}^{M}$ symmetric and strictly positive definite on $\mathbb{R}^{d} .\left(\tilde{V}_{t}^{M}, \tilde{Z}_{t}^{M}\right)$ will be the corresponding process replacing $\left(Z_{t}, V_{t}\right)$. For the time being we shall suppress the superscript $M$. Let

$$
\begin{aligned}
G^{*}= & \frac{1}{2} \sum \tilde{a}_{i j}(v) \frac{\partial^{2}}{\partial v_{i} \partial v_{j}}+\sum\left(\frac{\partial}{\partial v_{j}} \tilde{a}_{i j}(v)\right) \frac{\partial}{\partial v_{i}} \\
& -\sum \tilde{b}_{i}(v) \frac{\partial}{\partial v_{i}}-\sum v_{i} \frac{\partial}{\partial z_{i}}+\frac{1}{2} \sum \frac{\partial^{2}}{\partial v_{i} \partial v_{j}} \tilde{a}_{i j}(v)-\sum_{i} \frac{\partial}{\partial v_{i}} \tilde{b}_{i}(v)
\end{aligned}
$$

be the formal adjoint of the generator

$$
G=\frac{1}{2} \sum \tilde{a}_{i j}(v) \frac{\partial^{2}}{\partial v_{i} \partial v_{j}}+\sum \tilde{b}_{i}(v) \frac{\partial}{\partial v_{i}}+\sum v_{i} \frac{\partial}{\partial z_{i}}
$$

of the process $\left\{\tilde{V}_{t}, \tilde{Z}_{t}\right\}_{t \geqq 0}$. For fixed initial point $(w, y)$, let

$$
U(t, d v, d z \mid w, y)=P\left\{\tilde{V}_{t} \in d v, \tilde{Z}_{t} \in d z \mid V_{0}=w, Z_{0}=y\right\}
$$

be the distribution of $\left(\tilde{V}_{t}, \tilde{Z}_{t}\right)$. Then the formal density $u$ of $U$ is a distribution solution of the equation

$$
\left(-\frac{\partial}{\partial t}+G^{*}\right) u=0 \text { on }(0, \infty) \times \mathbb{R}^{d} \times \mathbb{R}^{d}
$$

More precisely, for any function $j \in C_{0}^{\infty}\left((0, \infty) \times \mathbb{R}^{d} \times \mathbb{R}^{d}\right)\left(C_{0}^{\infty}\right.$ denotes the $C^{\infty}$ functions with compact support)

$$
\int_{0}^{\infty} d t \int_{(v, z) \in \mathbb{R}^{d} \times \mathbb{R}^{d}} U(t, d v, d z)\left(\frac{\partial}{\partial t}+G\right) j(t, v, z)=0
$$

(see [9], p. 61). We now show that $-\partial / \partial t+G^{*}$ is hypoelliptic by means of Hörmander's theorem 1.1 in [19]. For this purpose let $\left(c_{i j}(v)\right)$ be the positive symmetric $C^{\infty}$ square root of $\tilde{a}_{i j}(v)$ (see [9], p. 83) and define the linear differential 
operators

$$
\begin{aligned}
X_{i}(v)= & \sum_{j} c_{i j}(v) \frac{\partial}{\partial v_{j}}, 1 \leqq i \leqq d, \\
X_{0}(v)= & -\frac{1}{2} \sum_{i, j, k} c_{i k}\left(\frac{\partial}{\partial v_{k}} c_{i j}\right) \frac{\partial}{\partial v_{j}}-\frac{\partial}{\partial t} \\
& +\sum_{i, j}\left(\frac{\partial}{\partial v_{j}} \tilde{a}_{i j}\right) \frac{\partial}{\partial v_{i}}-\sum_{i} \tilde{b}_{i} \frac{\partial}{\partial v_{i}}-\sum_{i} v_{i} \frac{\partial}{\partial z_{i}} .
\end{aligned}
$$

Then

$$
-\frac{\partial}{\partial t}+G^{*}=\frac{1}{2} \sum X_{i}^{2}+X_{0}+\frac{1}{2} \sum \frac{\partial^{2}}{\partial v_{i} \partial v_{j}} \tilde{a}_{i j}(v)-\sum_{i} \frac{\partial}{\partial v_{i}} \tilde{b}_{i}(v)
$$

A simple calculation verifies that each of the operators $\partial / \partial t, \partial / \partial v_{i}$ and $\partial / \partial z_{j}$ is a linear combination of the $X_{i}(v)$ or $\left[X_{i}(v), X_{j}(v)\right], 0 \leqq i, j \leqq d$. This is clear for the $\partial / \partial v_{i}$, because $c(v)=\tilde{a}^{1 / 2}(v)$ is nonsingular. Moreover

$$
\left[X_{i}, X_{0}\right]=-\sum_{k} c_{i, k}(v) \frac{\partial}{\partial z_{k}}+\text { linear combination of the } \frac{\partial}{\partial v_{i}}
$$

Thus also the $\partial / \partial z_{k}$ and hence $\partial / \partial t$ are in the Lie algebra generated by the $X_{i}$. By Theorem 1.1 of [17] this guarantees the hypoellipticity of $-\partial / \partial t+G^{*}$ and we conclude from (4.17) that we can write

$$
U(t, d v, d z \mid w, y)=u(t, v, z \mid w, y) d v d z
$$

for some $u$ which is in $C^{\infty}\left((0, \infty) \times \mathbb{R}^{d} \times \mathbb{R}^{d}\right)$ as a function of $(t, v, z)$. For our purposes it is necessary to have $u$ a continuous function of all its arguments $(t, v, z, u, y)$ on $(0, \infty) \times\left(\mathbb{R}^{d}\right)^{4}$. Following McKean $([9]$, p. 64) this can be done by combining the forward and the backward equation. $u$ (as a function of all its arguments) is a distribution solution of $K u=0$ on $(0, \infty) \times\left(\mathbb{R}^{d}\right)^{4}$, where

$$
K=-2 \frac{\partial}{\partial t}+G_{v, z}^{*}+G_{w, y}
$$

One shows as above that $K$ is hypoelliptic which gives the desired conclusion.

We need only a very weak consequence of the existence of a smooth density $u$. This is that for every $M, 0<\eta \leqq T, \lambda$ there exists a $K=K(M, \eta, T, \lambda)<\infty$ such that

$$
\begin{aligned}
& P\left\{\left|\tilde{Z}_{t+s}^{M}-z\right| \leqq \rho \mid V_{s}=w, Z_{s}=y\right\} \leqq K \rho^{d} \\
& 0 \leqq S \leqq T, \quad \eta \leqq t \leqq T, \quad|w|,|y|,|z| \leqq \lambda, \rho \leqq 1
\end{aligned}
$$

Now let $\varepsilon>0, T<\infty$, and choose $M=M(\varepsilon, T)$ such that

$$
P^{v_{0}}\left\{\left|V_{t}\right| \leqq \frac{1}{2 M} \text { or }\left|V_{t}\right| \geqq \frac{M}{2} \text { for some } t \leqq t\right\} \leqq \varepsilon
$$


For such an $M$ we have

$$
\begin{aligned}
P^{v_{0}} & \left\{\left|\int_{s}^{t} V_{\sigma} d \sigma\right|<\delta \text { for some } 0 \leqq s, t \leqq T \text { with }|t-s| \geqq \eta\right\} \\
\leqq & \varepsilon+P\left\{\int_{s}^{t} \tilde{V}_{\sigma}^{M} d \sigma<\delta \text { for some } 0 \leqq s, t \leqq T\right. \\
& \text { with } \left.|t-s|>\eta, \text { while } \frac{2}{M} \leqq\left|\widetilde{V}_{\sigma}\right| \leqq \frac{M}{2} \text { for all } \sigma \leqq T\right\} .
\end{aligned}
$$

This is so because if $V_{\sigma}$ is killed at the first time $\left|V_{\sigma}\right|$ leaves $(1 / M, M)$ and similarly for $\widetilde{V}_{\sigma}^{M}$, then the killed processes have the same distribution ([11], Chap. 5.24). Clearly

$$
\left|\int_{s}^{t} \tilde{V}_{\sigma}^{M} d \sigma\right| \leqq \frac{\delta}{4} \text { when }|t-s| \leqq \frac{\delta}{2 M},
$$

and

$$
\left|\int_{0}^{s} \widetilde{V}_{\sigma}^{M} d \sigma\right| \leqq T M, \quad s, t \leqq T,
$$

on the set $\left\{\left|\tilde{V}_{\sigma}^{M}\right| \leqq M / 2\right.$ for $\left.\sigma \leqq T\right\}$. We can use this to estimate the probability on the right-hand side of (4.19) by looking only at times which are multiples of $\rho=T / N$ for some integer $N$ with $\delta / 4 M \leqq T / N \leqq \delta / 2 M$. The probability in question is bounded by

$$
\begin{aligned}
& \sum_{\substack{0 \leqq i<j \leqq N \\
j-i \leqq \eta \rho^{-1}}} P\left\{\left|\int_{i \rho}^{j \rho} \tilde{V}_{\sigma}^{M} d \sigma\right| \leqq \frac{\delta}{2} \text { but }\left|\tilde{V}_{i \rho}^{M}\right| \leqq \frac{M}{2},\right. \\
& \text { and } \left.\left|\tilde{Z}_{i \rho}^{M}\right| \leqq T M\right\} \\
& \leqq N^{2} \max _{\substack{|t-s| \geqq \eta \\
0 \leqq s, t \leqq T \\
|w| \leqq M / 2,|y| \leqq T M}} P\left\{\left|\tilde{Z}_{t}^{M}-y\right| \leqq \frac{\delta}{2} \mid \tilde{V}_{s}^{M}=w, \tilde{Z}_{s}^{M}=y\right\} \\
& \leqq N^{2} K\left(\frac{\delta}{2}\right)^{d}(\text { by }(4.18)) \leqq K\left(\frac{4 M T}{\delta}\right)^{2}\left(\frac{\delta}{2}\right)^{d} .
\end{aligned}
$$

For $d \geqq 3$ the last expression tends to zero with $\delta$ so that $(4.2)$ follows from $(4,19)$.

Proof of Lemma 7. Apart from going over to the diffusion $W_{t} \equiv \Gamma V_{t}$ and letting the origin play the role of $\infty$, the proof of this lemma is in [9], Chap. 4.5. It is based on the observation that if $u$ is the positive function on $[0,1]$ which solves

$$
u\left(\frac{r^{2}}{2}\right)=1+2 \int_{r}^{1} \frac{t}{C_{-}(t)} d t \int_{t}^{1} \frac{C_{-}(s)}{A_{+}(s)} s u\left(\frac{s^{2}}{2}\right) d s,
$$

then

$$
Y_{t} \equiv e^{-t \wedge \tau} u\left(\left|\Gamma V_{t \wedge \tau}\right|^{2} / 2\right)
$$


is a positive supermartingale, where $\tau=\inf \left\{t \geqq 0:\left|V_{t}\right| \geqq 1\right\}$, and on the fact that $u(r) \rightarrow \infty$ as $r \downarrow 0$ if (4.4) holds. (We have $C_{-}$in contrast to $C_{+}$in [9] because our $u$ is decreasing rather than increasing).

Example a. We already remarked that if $r_{i j}$ in (4.5) vanishes for $|y| \geqq L$, then (2.2) trivially holds. If we take in addition $r_{i j}$ of the form (4.6) with $\rho(y, v)=\rho^{*}(|y|)$ then $a_{i j}$ and $b_{i}$ of (2.3) and (2.4) become

$$
\begin{aligned}
a_{i j}(v) & =\int_{-\infty}^{+\infty} d t r_{i j}(t v, v, v) \\
& =\sum_{k} \int_{-\infty}^{+\infty} d t \int_{\mathbb{R}^{d}} \rho_{i k}^{*}(|t v+z|) \overline{\rho_{j k}^{*}}(|z|) d z \\
& =\frac{1}{|v|} \sum_{k}^{\infty} \int_{0}^{\infty} d t \int_{\mathbb{R}^{d}} \rho_{i k}^{*}(|t e+z|) \overline{\rho_{j k}^{*}}(|z|) d z, \\
b_{i}(v) & =\frac{1}{2} \sum_{j} \frac{\partial}{\partial v_{j}} a_{i j}(v) .
\end{aligned}
$$

In this case $\mathscr{L}$ has the form (4.10). With this $\mathscr{L}(2.6)$ is valid by Remark 6. Condition (IV) can be verified in the same way as for the Gaussian examples of [6], Theorem 4, since (4.8) and (4.9) assure that

$$
\begin{aligned}
& \left(\frac{\partial}{\partial y_{k}}\right)^{2}\left(\frac{\partial}{\partial y_{l}}\right)^{2} r_{i i}(y) \text { exists, and } \\
& \left|\left(\frac{\partial}{\partial y_{k}}\right)^{2}\left(\frac{\partial}{\partial y_{l}}\right)^{2} r_{i i}(0)-\left(\frac{\partial}{\partial y_{k}}\right)^{2}\left(\frac{\partial}{\partial y_{l}}\right)^{2} r_{i i}(u)\right|=O\left(\log \frac{\partial}{|u|}\right)^{\alpha} .
\end{aligned}
$$

Example b. It is clear that any field $F$ of the form (4.11), (4.12) is stationary in $x$, because of the translation invariance of the point process $P_{\rho}$. It is also clear that the randomness in $K(x)$ and hence in $F(x, v)$ depends only on the position of the points $p_{n}$ within distance $L$ of $x$ and the corresponding $H^{(n)}$. This is so because all $H^{(n)}(x)$ vanish for $|x| \geqq L$. In particular $F\left(x_{1}, v_{1}\right)$ and $F\left(x_{2}, v_{2}\right)$ will be independent when $\left|x_{1}-x_{2}\right|>2 L$. More generally $\mathscr{G}_{\Lambda_{1}}$ and $\mathscr{G}_{\Lambda_{2}}$ will be independent when $d\left(\Lambda_{1}, \Lambda_{2}\right)>2 L$ and hence $\beta(\rho)=0$ for $\rho>2 L$. Formula (2.2) is again immediate.

Let us now specialize to (4.15) with the distribution of $H^{(0)}\left(x_{1}\right)$ and $H^{(0)}\left(x_{2}\right)$ invariant under the change $x_{i} \rightarrow O x_{i}$, and (4.13), (4.14). Clearly, $E\{F(x)\}=0$ by (4.13), so that (II) holds. (IV) is easily derived from (4.14), and (I) holds for $F$ because the $H^{(n)}(x)$ are assumed to be $C^{2}\left(\mathbb{R}^{d}\right)$. As for $(\mathrm{V})$, the coefficients $a_{i j}(v)$ and $b_{i}(i)$ of $(2.3)$ and (2.4) now are

$$
\begin{aligned}
a_{i j}(v) & =\int_{-\infty}^{+\infty} d t \sum_{n, m} E\left\{H_{i}^{(n)}\left(p_{n}\right) H_{j}^{(m)}\left(t v+p_{m}\right)\right\} \\
& =\int_{-\infty}^{+\infty} d t \sum_{n} E\left\{H_{i}^{(n)}\left(p_{n}\right) H_{j}^{(n)}\left(t v+p_{n}\right)\right\} \\
& =\int_{-\infty}^{+\infty} d t \int_{\mathbb{R}^{d}} \rho d z E\left\{H_{i}^{(0)}(z) H_{j}^{(0)}(t v+z)\right\}
\end{aligned}
$$




$$
=\frac{2 \rho}{|v|} \int_{0}^{\infty} d t \int_{\mathbb{R}^{d}} E\left\{H_{i}^{(0)}(z) H_{j}^{(0)}(t e+z)\right\} d z .
$$

$(v=|v|$ Oe for some orthogonal $O)$, and

$$
b_{i}(v)=\frac{1}{2} \sum_{j} \frac{\partial}{\partial v_{j}} a_{i j}(v) .
$$

This $\mathscr{L}$ is given by (4.6) and (2.6) is again guaranteed by Remark 6.

\section{References}

1. Kubo, R. : Stochastic Liouville equation. J. Math. Phys. 4, 174-183 (1963)

2. Van Kampen, N. G. : Stochastic differential equations. Phys. Rep. (Section C Phys. Lett.) 24, $171-228(1976)$

3. Sturrock, P. A. : Stochastic acceleration. Phys. Rev. 141, 186-191 (1966)

4. Hall, D. E. Sturrock, P. A. : Diffusion, scattering and acceleration of particles by Stochastic electromagnetic fields. Phys. Fluids 10, 2620-2628 (1967).

5. Silevitch, M.B., Golden, K. I. : Dielectric formulation of test particle energy loss in a plasmas. J. Stat. Phys. 7, 65-87 (1973)

6. Kesten, H. Papanicolaou, G. C. : A limit theorem for turbulent diffusion. Commun. Math. Phys. 65, 97-128 (1979)

7. Ibragimov, I. A., Linnik, Yu. V. : Independent and stationary sequences of random variables. Groningen: Walters-Noordoff 1971

8. Billingsley, P. : Convergence of probability measures. New York: Wiley, 1968

9. McKean Jr., H. P. : Stochastic integrals. New York: Academic Press 1969

10. Nelson, E. : An existence theorem for second order parabolic equations. Trans. Am. Math. Soc. 88, 414-429 (1958)

11. Dynkin, E. B. : Markov processes I and II. Berlin, Heidelberg, New York: Springer 1965

12. Stone, C.: Weak convergence of stochastic processes defined on semi-infinite time intervals, Proc. Am. Math. Soc. 14, 694-696 (1963)

13. Lindval, T. : Weak convergence of probability measures and random functions in the function space D [0, $\infty)$, J. Appl. Prop. 10, 109-121 (1973)

14. Meyer, P. A. : Probability and potentials. New York: Blaisdell 1966

15. Stroock, D. W., Varadhan, S. R. S. : Diffusion processes with boundary conditions. Commun. Pure Appl. Math. 24, 147-225 (1971)

16. Stroock, D. W., Varadhan, S. R. S. : Diffusion processes with continuous coefficients I. Commun. Pure Appl. Math. 22, 345-400 (1969)

17. Stroock, D. W., Varadhan, S. R. S. : On the support of diffusion processes with applications to the strong maximum principle. Proc. 6th Berkeley Symp. Math. Statist. Prob., Vol. II, pp. 339-359. Univ. of California Press 1972

18. Khasminskii, R. Z. : Ergodic properties of recurrent diffusion processes and stabilization of the solution of the Cauchy problem for parabolic equations. Th. Prob. Appl. 5, 179-196 (1960)

19. Hörmander. L. : Hypoelliptic second order differential equations. Acta Math. 119, 147-171 (1967)

Communicated by J. Lebowitz

Received December 19,1979 
